\title{
Self-dual chiral boson: augmented superfield approach
}

\author{
D. Shukla ${ }^{1, a}$, T. Bhanja ${ }^{1, b}$, R. P. Malik ${ }^{1,2, c}$ \\ ${ }^{1}$ Department of Physics, Faculty of Science, Centre of Advanced Studies, Banaras Hindu University, Varanasi 221 005, U.P., India \\ ${ }^{2}$ Faculty of Science, DST Centre for Interdisciplinary Mathematical Sciences, Banaras Hindu University, Varanasi 221 005, U.P., India
}

Received: 29 April 2014 / Accepted: 6 August 2014 / Published online: 16 September 2014

(C) The Author(s) 2014. This article is published with open access at Springerlink.com

\begin{abstract}
We exploit the standard tools and techniques of the augmented version of the Bonora-Tonin superfield formalism to derive the off-shell nilpotent and absolutely anticommuting (anti-)Becchi-Rouet-Stora-Tyutin (BRST) and (anti-)co-BRST symmetry transformations for the BRSTinvariant Lagrangian density of a self-dual bosonic system. In the derivation of the full set of the above symmetry transformations, we invoke the (dual-)horizontality conditions, and (anti-)BRST- and (anti-)co-BRST-invariant restrictions on the superfields that are defined on the $(2,2)$-dimensional supermanifold. The latter is parameterized by the bosonic variable $x^{\mu}(\mu=0,1)$ and a pair of Grassmannian variables $\theta$ and $\bar{\theta}$ (with $\theta^{2}=\bar{\theta}^{2}=0$ and $\theta \bar{\theta}+\bar{\theta} \theta=0$ ). The dynamics of this system is such that, instead of the full $(2,2)$ dimensional superspace coordinates $\left(x^{\mu}, \theta, \bar{\theta}\right)$, we require only the specific $(1,2)$-dimensional super-subspace variables $(t, \theta, \bar{\theta})$ for its description. This is a novel observation in the context of the superfield approach to the BRST formalism. The application of the dual-horizontality condition, in the derivation of a set of proper (anti-)co-BRST symmetries, is also one of the new ingredients of our present endeavor where we have exploited the augmented version of the superfield approach to the BRST formalism.
\end{abstract}

\section{Introduction}

The model of a self-dual chiral boson is a widely studied subject (see, e.g., [1-12]) because it finds its use in the description of models of superstrings, quantum Hall effect, $\mathrm{W}$-gravities, and some of the two dimensional statistical systems. In a recent paper [13], this model has also been shown to be a field-theoretical example for the Hodge theory because this two $(1+1)$-dimensional $(2 \mathrm{D})$ system provides physi-

\footnotetext{
a e-mail: dheerajkumarshukla@gmail.com

be-mail: tapobroto.bhanja@gmail.com

c e-mail: malik@bhu.ac.in
}

cal realizations of the de Rham cohomological operators of differential geometry in the language of its symmetry properties (and corresponding conserved charges). The purpose of our present investigation is to apply the augmented version of the Bonora-Tonin (BT)-superfield formalism [1419] to derive its proper nilpotent (anti-)Becchi-Rouet-StoraTyutin (BRST) and (anti-)co-BRST symmetry transformations (which together lead to the derivation of a unique bosonic symmetry transformation [13]). These symmetries collectively lead to the physically conceivable realizations of the cohomological operators.

The above superfield approach to the BRST formalism is one of the geometrically intuitive methods that shed light on the abstract mathematical properties associated with the proper (anti-)BRST symmetries in the language of geometrical objects on the supermanifold. Usually, within the framework of this superfield approach, a given D-dimensional ordinary gauge theory is generalized to a $(\mathrm{D}, 2)$-dimensional supermanifold where the superfields are defined corresponding to the ordinary dynamical fields of a given ordinary gauge theory. It is the $(\mathrm{D}, 2)$-dimensional superspace coordinates ${ }^{1}$ $Z^{M}=\left(x^{\mu}, \theta, \bar{\theta}\right)$ that characterize the (D, 2)-dimensional supermanifold. The gauge-invariant restrictions on the superfields lead to the derivation of (anti-)BRST symmetry transformations. On the other hand, the translational generators $\left(\partial_{\theta}, \partial_{\bar{\theta}}\right)$, along the Grassmannian directions $(\theta, \bar{\theta})$, provide the geometrical basis for the (anti-)BRST symmetries and the corresponding conserved charges.

One of the decisive features of our present investigation is the key observation that it is the super-subspace coordinates that are good enough to define the super exterior derivative and the supergauge connection in the description of the $2 \mathrm{D}$ self-dual chiral bosonic system within the framework of the

\footnotetext{
${ }^{1}$ In the definition of $Z^{M}=\left(x^{\mu}, \theta, \bar{\theta}\right)$, the ordinary coordinates $x^{\mu}$ (with $\mu=0,1,2, \ldots, D-1$ ) are the bosonic variables of the Ddimensional ordinary gauge theory and the pair of coordinates $\theta$ and $\bar{\theta}$ are the Grassmannian variables with their fermionic properties: $\theta^{2}=$ $\bar{\theta}^{2}=0, \theta \bar{\theta}+\bar{\theta} \theta=0$.
} 
superfield formalism. In other words, even though we require a $(2,2)$-dimensional supermanifold to define the superfields (corresponding to the 2D ordinary fields of the self-dual chiral boson), the (dual-)horizontality conditions require $(1,2)$ dimensional super-subspace variables for their description (see, e.g., Sects. 3, 6). This peculiarity arises because of the fact that only one component of the "gauge" field (i.e. $\lambda=\lambda_{0}+\lambda_{1}$ ) is the dynamical as well as propagating field and its orthogonal counterpart $\left(\lambda_{0}-\lambda_{1}\right)$ remains inert. This is a novel observation in the superfield approach to a given gauge theory (when the latter is discussed within the framework of the BRST formalism). Stated explicitly, we note that our superfields, corresponding to the $2 \mathrm{D}$ ordinary fields of the chiral boson model, would be a function of the $(2,2)$ dimensional superspace variables $Z^{M}=\left(x^{\mu}, \theta, \bar{\theta}\right)$. However, the super exterior derivative $\tilde{d}$ and the super 1-form connection $\tilde{A}^{(1)}$ (which play important roles in the restrictions on the appropriate superfields) would be defined in terms of the $(1,2)$-dimensional super-subspace variables $(t, \theta, \bar{\theta})$ [cf. (19) below].

It is the above cited novel feature that has propelled us to pursue our present investigation within the framework of our augmented version of the BT-superfield formalism so that we could derive the (anti-)BRST and (anti-)co-BRST symmetry transformations of this theory as well as provide the geometrical interpretations for them. One of the key signatures of a gauge theory is the existence of (anti-)BRSTinvariant Curci-Ferrari (CF) type restrictions [20] when this gauge theory is discussed within the framework of the superfield approach to the BRST formalism. For our 2D self-dual chiral bosonic system, we find that the CF-type conditions are trivial and they are found to be (anti-)BRST as well as (anti-)co-BRST invariant, respectively [in the context of (anti-)BRST and (anti-)co-BRST symmetries]. Ultimately, in our present endeavor, we obtain the proper (i.e. nilpotent and absolutely anticommuting) (anti-)BRST and (anti-)co-BRST symmetries for the 2D system under consideration and provide the geometrical basis for the symmetries and conserved charges. It is gratifying to state that we have checked that our proposal for the Hodge-duality operation on the $(1,2)$ dimensional super-subspace differentials (and their wedge products) turns out to be correct and it leads to the derivation of proper (anti-)co-BRST symmetries and (anti-)co-BRSTinvariant CF-type condition. The latter is a decisive feature of the BRST approach to a gauge theory.

As a background of our further discussions, we state a few facts about the 2D self-dual chiral bosonic model. To start with, the covariant version of the self-dual chiral bosonic system is non-gauge invariant but, after the inclusion of the Wess-Zumino term, it becomes a gauge-invariant theory (see, e.g., Sect. 2). In other words, the inclusion of the WessZumino term converts the second-class constraints of the original theory into their counterpart first-class constraints (thereby rendering the theory gauge invariant). To corroborate the above statements, let us begin with the Lorentz covariant version of Lagrangian density (see e.g. [21]) of the self-dual chiral boson in two $(1+1)$ dimensions of spacetime $^{2}$

$\mathcal{L}_{(0)}=\frac{1}{2} \partial_{\mu} \phi \partial^{\mu} \phi+\lambda_{\mu}\left(\varepsilon^{\mu \nu}+\eta^{\mu \nu}\right) \partial_{\nu} \phi$,

where the scalar field $\phi$ obeys the self-duality condition (i.e. $\dot{\phi}=\phi^{\prime}$ ) due to the following Euler-Lagrange (EL) equation of motion:

$\left(\varepsilon^{\mu v}+\eta^{\mu v}\right) \partial_{\nu} \phi=0 \quad \Longrightarrow \quad \dot{\phi}=\phi^{\prime}$.

The other covariant form of the EL equation of motion,

$\square \phi+\left(\varepsilon^{\mu \nu}+\eta^{\mu \nu}\right) \partial_{\nu} \lambda_{\mu}=0$,

implies the masslessness condition $\square \phi=0$ and the selfduality condition $\dot{\lambda}=\lambda^{\prime}$ where $\lambda=\lambda_{0}+\lambda_{1}$. Thus, it is clear that there are two self-dual fields (i.e. $\dot{\phi}=\phi^{\prime}, \dot{\lambda}=\lambda^{\prime}$ ) in our theory at the classical level. The non-Lorentz covariant form of a single chiral boson was written in [8], which was given in Lorentz covariant form in a couple of very interesting papers [22,23]. It can be checked that, in the component form [with $\eta_{\mu \nu}=\operatorname{diag}(+1,-1)=\eta^{\mu \nu}$ ], we have the following expression for this starting Lagrangian density (1):

$\mathcal{L}_{(0)}=\frac{1}{2}\left(\dot{\phi}^{2}-\phi^{\prime 2}\right)+\lambda\left(\dot{\phi}-\phi^{\prime}\right)$,

which is normally used for the description of the self-dual chiral boson. ${ }^{3}$ We re-emphasize that it is the combination $\lambda=\lambda_{0}+\lambda_{1}$ that participates in the description of the selfdual chiral boson but its orthogonal counterpart $\left(\lambda_{0}-\lambda_{1}\right)$ does not appear in the Lagrangian density. In our further discussions, we shall focus only on $\left(\lambda=\lambda_{0}+\lambda_{1}\right)$ as the propagating "gauge" field (see, e.g., [21] for details) and shall completely ignore the non-propagating $\left(\lambda_{0}-\lambda_{1}\right)$ component (see Sect. 3 below) of the gauge field.

Our present investigation is essential on the following counts. First, the self-dual chiral boson is an interesting model, which is required in many physical systems of importance. Thus, anything novel about this model is interesting in itself. We observe some novelties in the application of the augmented version of superfield approach to the BRST formalism for this system. As it turns out, we need only

\footnotetext{
2 We adopt here the convention and notations such that the 2D flat Minkowski spacetime is endowed with a flat metric with signatures $(+1,-1)$ and the 2D Levi-Civita tensor is chosen to satisfy $\varepsilon_{\mu \nu} \varepsilon^{\mu \nu}=$ $-2 !, \varepsilon_{\mu \nu} \varepsilon^{\mu \lambda}=-\delta_{\mu}^{\lambda}$ with $\varepsilon_{01}=+1=-\varepsilon^{01}$. The overdot and prime on the fields, throughout the text, always correspond to the partial derivatives w.r.t. time and space variables, respectively.

3 At this stage, this theory is non-gauge invariant. However, after the inclusion of the Wess-Zumino term, it becomes gauge invariant (see, e.g., Sect. 2 below).
} 
$(1,2)$-dimensional super-subspace variables for its characterization. Second, it was challenging for us to apply the theoretical arsenal of the augmented version of the superfield formalism to this system where only one component $\left(\lambda=\lambda_{0}+\lambda_{1}\right)$ of the gauge field couples with the matter field and its orthogonal component remains in the background. In our earlier applications of the superfield formalism (see, e.g., [16-19]) to (non-)Abelian gauge theories, we have never come across the peculiarity of the 2D chiral bosonic system under consideration. Finally, it was very important for us to apply and check the sanctity of the Hodge-duality operation on the $(2,2)$-dimensional supermanifold in the determination of (anti-)co-BRST symmetries that are present in the theory. As it turns out for our present theory, we need only $(1,2)$-dimensional super-subspace variables $(t, \theta, \bar{\theta})$ for its description (and the Hodge duality is applied on these coordinates only). We have accomplished all the above goals in our present endeavor.

The contents of our present endeavor are organized as follows. In Sect. 2, we discuss the bare essentials of the (anti-)BRST symmetry transformations (and corresponding conserved charges) for the self-dual chiral boson in the Lagrangian formulation. Section 3 is devoted to the derivation of the above (anti-)BRST transformations using the augmented version of the BT-superfield formalism. In Sect. 4, we deal with the proof of the (anti-)BRST invariance of the super Lagrangian density and capture the nilpotency and absolute anticommutativity properties of the (anti-)BRST charges within the framework of our superfield approach. Section 5 contains a brief synopsis of the (anti-)co-BRST symmetries (and corresponding charges) of our present theory. We derive these symmetries in Sect. 6 using the superfield formalism. Section 7 deals with the (anti-)co-BRST invariance of the Lagrangian density and nilpotency of the (anti-)co-BRST charges within the framework of our superfield approach. Finally, we make some concluding remarks and point out a few future directions for further investigations in Sect. 8.

In Appendix A, we discuss the key differences between the nilpotent (anti-)BRST and (anti-)co-BRST symmetries and our Appendices B and C are devoted to the explicit computations of (i) the dual-horizontality condition, and (ii) the auxiliary variable $\mathcal{B}$.

\section{Preliminary: nilpotent (anti-)BRST symmetries in Lagrangian formulation}

Let us begin with the (2D) second-order Lagrangian density $\left(\mathcal{L}_{b}\right)$ for the description of a single self-dual chiral boson within the framework of BRST formalism. This effective Lagrangian density of the 2D chiral boson (with the inclusion of the Wess-Zumino term) is as follows (see, e.g., [13,24] for details):

$$
\begin{aligned}
\mathcal{L}_{b}= & \frac{\dot{\phi}^{2}}{2}-\frac{\dot{v}^{2}}{2}+\dot{v}\left(v^{\prime}-\phi^{\prime}\right)+\lambda\left[\dot{\phi}-\dot{v}+v^{\prime}-\phi^{\prime}\right] \\
& -\frac{1}{2}\left(\phi^{\prime}-v^{\prime}\right)^{2}+B(\dot{\lambda}-v-\phi) \\
& +\frac{B^{2}}{2}-i \dot{\bar{C}} \dot{C}+2 i \bar{C} C, \\
\equiv & \frac{\dot{\phi}^{2}}{2}-\frac{1}{2}\left[\dot{v}-\left(v^{\prime}-\phi^{\prime}\right)\right]^{2}+\lambda\left[\dot{\phi}-\dot{v}+v^{\prime}-\phi^{\prime}\right] \\
& +B(\dot{\lambda}-v-\phi)+\frac{B^{2}}{2}-i \dot{\bar{C}} \dot{C}+2 i \bar{C} C,
\end{aligned}
$$

where $\dot{\phi}=\partial \phi / \partial t, \dot{v}=\partial v / \partial t, \dot{\bar{C}}=\partial \bar{C} / \partial t, \dot{\lambda}=\partial \lambda /$ $\partial t$, etc, are the generalized "velocities" with respect to the evolution parameter $t, B$ is the Nakanashi-Lautrup auxiliary field, the fermionic $\left(C^{2}=\bar{C}^{2}=0, C \bar{C}+\bar{C} C=0\right)$ (anti-)ghost fields $(\bar{C}) C$ are required for the validity of unitarity in our theory and the notations $\phi^{\prime}=(\partial \phi / \partial x), v^{\prime}=(\partial v /$ $\partial x$ ) stand for the single space derivative on the $2 \mathrm{D}$ chiral boson field $\phi$ and Wess-Zumino (WZ) field $v$, respectively. We further note that $B(\dot{\lambda}-v-\phi)+\frac{1}{2} B^{2}=-\frac{1}{2}(\dot{\lambda}-$ $v-\phi)^{2}$ is the gauge-fixing term for the gauge field $\lambda(x)$ where $B=-(\dot{\lambda}-v-\phi)$. The above Lagrangian density is the (anti-)BRST-invariant generalization of (4) and it respects the following off-shell nilpotent and infinitesimal (anti-)BRST symmetry transformations $\left(s_{(a) b}\right)$ :

$s_{a b} \phi=-\bar{C}, \quad s_{a b} v=-\bar{C}, \quad s_{a b} \lambda=\dot{\bar{C}}, \quad s_{a b} \bar{C}=0$,

$s_{a b} C=-i B, \quad s_{a b} B=0$,

$s_{b} \phi=-C, \quad s_{b} v=-C, \quad s_{b} \lambda=\dot{C}, \quad s_{b} \bar{C}=+i B$,

$s_{b} C=0, \quad s_{b} B=0$.

In fact, it can be explicitly checked that the (anti-)BRSTinvariant Lagrangian density (5) transforms, under $s_{(a) b}$, to the total time derivatives as

$s_{b} \mathcal{L}_{b}=\frac{\partial}{\partial t}[B \dot{C}], \quad s_{a b} \mathcal{L}_{b}=\frac{\partial}{\partial t}[B \dot{\bar{C}}]$.

Thus, the action integral $S=\int \mathrm{d} x \int \mathrm{d} t \mathcal{L}_{b}$ remains invariant under the above (anti-)BRST transformations. Further, it is straightforward to note that the above (anti-)BRST symmetry transformations (6) are off-shell nilpotent $\left(s_{b}^{2}=s_{a b}^{2}=0\right)$ and absolutely anticommuting $\left(s_{b} s_{a b}+s_{a b} s_{b}=0\right)$ in nature without any use of the Euler-Lagrange (EL) equations of motion:

$$
\begin{aligned}
\dot{B}= & \dot{\phi}-\dot{v}+v^{\prime}-\phi^{\prime}, \quad B=v+\phi-\dot{\lambda}, \quad \ddot{C}+2 C=0, \\
& -B=\ddot{\phi}+\dot{\lambda}-\dot{v}^{\prime}-\lambda^{\prime}-\left(\phi^{\prime \prime}-v^{\prime \prime}\right), \quad \ddot{\bar{C}}+2 \bar{C}=0, \\
B= & \ddot{v}-2 \dot{v}^{\prime}+\dot{\phi}^{\prime}+\dot{\lambda}-\lambda^{\prime}-\left(\phi^{\prime \prime}-v^{\prime \prime}\right),
\end{aligned}
$$

which emerge from the Lagrangian density (5). The off-shoot of the above equations is the observation that $\ddot{B}+2 B=0$, because $(-2 B)=\ddot{\phi}-\ddot{v}+\dot{v}^{\prime}-\dot{\phi}^{\prime}$, which is equal to $\ddot{B}$ (as is evident from the first entry of the above equations: 
$\dot{B}=\dot{\phi}-\dot{v}+v^{\prime}-\phi^{\prime}$ ). We shall see, later on, that this relation is something like the Curci-Ferrari type restriction [20] because it plays an important role in proving the anticommutativity property.

By exploiting the standard tricks and techniques of Noether's theorem, it can be checked that the conserved $\left(\dot{Q}_{(a) b}=0\right)$, nilpotent $\left(Q_{(a) b}^{2}=0\right)$, and absolutely anticommuting ( $\left.Q_{b} Q_{a b}+Q_{a b} Q_{b}=0\right)$ (anti-)BRST charges: ${ }^{4}$

$$
\begin{aligned}
Q_{b} & =\int \mathrm{d} x\left[B \dot{C}-\left(\dot{\phi}-\dot{v}+v^{\prime}-\phi^{\prime}\right) C\right] \\
& \equiv \int \mathrm{d} x[B \dot{C}-\dot{B} C], \\
Q_{a b} & =\int \mathrm{d} x\left[B \dot{\bar{C}}-\left(\dot{\phi}-\dot{v}+v^{\prime}-\phi^{\prime}\right) \bar{C}\right] \\
& \equiv \int \mathrm{d} x[B \dot{\bar{C}}-\dot{B} \bar{C}]
\end{aligned}
$$

are the generators of the (anti-)BRST symmetry transformations (6). Using this property, it is straightforward to prove the following relationships:

$s_{b} Q_{b}=-i\left\{Q_{b}, Q_{b}\right\}=0$,

$s_{a b} Q_{a b}=-i\left\{Q_{a b}, Q_{a b}\right\}=0$,

$s_{b} Q_{a b}=-i\left\{Q_{a b}, Q_{b}\right\}=0$,

$s_{a b} Q_{b}=-i\left\{Q_{b}, Q_{a b}\right\}=0$,

which demonstrate the nilpotency and absolute anticommutativity property of the conserved (anti-)BRST charges $Q_{(a) b}$. In the proof of the conservation of the charges $Q_{(a) b}$, one has to exploit the off-shoot of the equation of motion (8), which shows that $\ddot{B}+2 B=0$. In the forthcoming two subsequent sections, we shall capture these properties within the framework of our augmented version of the BT-superfield approach.

We end this section with the remark that there exist global infinitesimal ghost-scale symmetry transformations ${ }^{5} s_{g} C=$ $C, s_{g} \bar{C}=-\bar{C}, s_{g} \Psi=0,(\Psi=\phi, v, \lambda)$ in the theory which are generated by the Noether conserved ghost charge $Q_{g}=-i \int \mathrm{d} x[(C \dot{\bar{C}}+\bar{C} \dot{C})]$. It is elementary to check that the ghost charge $Q_{g}$ satisfies the following algebra with the nilpotent and conserved (anti-)BRST charges:

$i\left[Q_{g}, Q_{b}\right]=Q_{b}, \quad i\left[Q_{g}, Q_{a b}\right]=-Q_{a b}$.

\footnotetext{
4 Our expressions for the off-shell nilpotent (anti-)BRST charges match with [24] but we have a slight difference from the corresponding expressions quoted in [13] at the conceptual level.

5 To be precise, the fields of our present theory transform as $\Psi \rightarrow$ $\mathrm{e}^{0 \Omega} \Psi, C \rightarrow \mathrm{e}^{1 \Omega} C, \bar{C} \rightarrow \mathrm{e}^{-1 \Omega} \bar{C}$ where $\Omega$ is a global scale infinitesimal transformation parameter and the generic field $\Psi=\phi, v, \lambda$. The numerals in the exponents denote the ghost number of the fields. We set $\Omega=1$ to obtain the simpler form of the infinitesimal version of these transformations as: $s_{g} C=C, s_{g} \bar{C}=-\bar{C}, s_{g} \Psi=0$, and the algebra $s_{g} Q_{b}=-i\left[Q_{b}, Q_{g}\right]=+Q_{b}, s_{g} Q_{a b}=-i\left[Q_{a b}, Q_{g}\right]=-Q_{a b}$.
}

The above algebra, together with the algebra quoted in (10), obeys the standard BRST algebra amongst the nilpotent (anti-)BRST charges $Q_{(a) b}$ and the ghost charge $Q_{g}$, namely

$\left\{Q_{(a) b}, Q_{(a) b}\right\}=Q_{(a) b}^{2}=0, \quad\left\{Q_{b}, Q_{a b}\right\}=0$,

$i\left[Q_{g}, Q_{b}\right]=Q_{b}, \quad i\left[Q_{g}, Q_{a b}\right]=-Q_{a b}$,

which establishes that the ghost number of the BRST charge is $(+1)$ and that of the anti-BRST charge is $(-1)$ and the (anti-)BRST charges are nilpotent of order two. We further note that one can derive the on-shell nilpotent (anti-)BRST symmetry transformations from (6) by replacing $B=-(\dot{\lambda}-$ $v-\phi)$. These symmetry transformations are:

$s_{a b} \phi=-\bar{C}, \quad s_{a b} v=-\bar{C}, \quad s_{a b} \lambda=\dot{\bar{C}}$,

$s_{a b} \bar{C}=0, \quad s_{a b} C=+i(\dot{\lambda}-v-\phi)$,

$s_{b} \phi=-C, \quad s_{b} v=-C, \quad s_{b} \lambda=\dot{C}$,

$s_{b} C=0, \quad s_{b} \bar{C}=-i(\dot{\lambda}-v-\phi)$.

For the sake of brevity, we have adopted the same notations for the off-shell as well as on-shell (anti-)BRST symmetry transformations. We further observe that

$s_{a b}^{2} C=-i[\ddot{\bar{C}}+2 \bar{C}], \quad s_{b}^{2} \bar{C}=+i[\ddot{C}+2 C]$.

The terms of the r.h.s. of the above transformations are zero on-shell where the equations of motion $\ddot{\bar{C}}+2 \bar{C}=$ $0, \quad \ddot{C}+2 C=0$ are valid. Similarly, we point out that the absolute anticommutativity property of (13) is satisfied if and only if (8) are taken into account. The above on-shell nilpotent symmetry transformations are true symmetry transformations for the following Lagrangian density (without the presence of the Nakanishi-Lautrup field $B$ ):

$$
\begin{aligned}
\mathcal{L}_{b}^{(0)}= & \frac{\dot{\phi}^{2}}{2}-\frac{\dot{v}^{2}}{2}+\dot{v}\left(v^{\prime}-\phi^{\prime}\right)+\lambda\left[\dot{\phi}-\dot{v}+v^{\prime}-\phi^{\prime}\right] \\
& -\frac{1}{2}\left(\phi^{\prime}-v^{\prime}\right)^{2}-\frac{1}{2}(\dot{\lambda}-v-\phi)^{2}-i \dot{\bar{C}} \dot{C} \\
& +2 i \bar{C} C,
\end{aligned}
$$

because we have the following transformations:

$s_{b} \mathcal{L}_{b}^{(0)}=-\frac{\partial}{\partial t}[(\dot{\lambda}-v-\phi) \dot{C}]$,

$s_{a b} \mathcal{L}_{b}^{(0)}=-\frac{\partial}{\partial t}[(\dot{\lambda}-v-\phi) \dot{\bar{C}}]$.

The above observations demonstrate that the action integral $S=\int \mathrm{d}^{2} x \mathcal{L}_{b}^{(0)}$ remains invariant under the on-shell nilpotent symmetry transformations (13). The expressions for the onshell nilpotent conserved charges $Q_{(a) b}$ can be derived from (9) by the replacements $B=-(\dot{\lambda}-v-\phi) \equiv-\frac{1}{2}[(\ddot{\phi}-$ $\left.\ddot{v})-\left(\dot{\phi}^{\prime}-\dot{v}^{\prime}\right)\right]$ and $\dot{B}=(\dot{\phi}-\dot{v})-\left(\phi^{\prime}-v^{\prime}\right)$. The stage is now set to discuss the original theory (1) at the quantum level. In this connection, we note that our original theory (1) has been modified due to the presence of the WZ field 
$v$. Within the framework of BRST formalism, the physical state $\mid$ phys $>$ of the theory is defined as $Q_{(a) b} \mid$ phys $>=0$ at the quantum level. Using the expressions for $Q_{(a) b}$ from (9), it is clear that $B \mid$ phys $>=0, \dot{B} \mid$ phys $>=0$, due to the fact that the (anti-)ghost fields are decoupled from the rest of the theory and they themselves do not produce any physically meaningful constraints on the theory. From the EL equations of motion (8), it is clear that the above conditions imply that $\left[(\dot{\phi}-\dot{v})-\left(\phi^{\prime}-v^{\prime}\right)\right] \mid$ phys $>=0$ and $[(\ddot{\phi}-\ddot{v})-$ $\left.\left(\dot{\phi}^{\prime}-\dot{v}^{\prime}\right)\right] \mid$ phys $>=0$. With the redefinition $\tilde{\phi}=(\phi-v)$, it is evident that, ultimately, we have the conditions on the physical state $\left(\dot{\tilde{\phi}}-\tilde{\phi}^{\prime}\right) \mid$ phys $>=0$ and $\frac{\mathrm{d}}{\mathrm{d} t}\left(\dot{\tilde{\phi}}-\tilde{\phi}^{\prime}\right) \mid$ phys $>$ $=0$, which imply the self-duality condition and its timeevolution invariance. ${ }^{6}$ Thus, at the quantum level, in reality, there exists only a single self-dual chiral boson $\phi$ and the duality condition $\dot{\lambda}=\lambda^{\prime}$ does not appear at the quantum level in any physically meaningful limit.

We shall concentrate on the Lagrangian density (15) for our further discussions on the (anti-)dual-BRST symmetry transformations (Sect. 5).

\section{3 (Anti-)BRST symmetries: superfield formalism}

To derive the (anti-)BRST symmetry transformations (6) within the framework of the augmented superfield formalism, first of all, we generalize the basic variables of the $2 \mathrm{D}$ theory onto the $(2,2)$-dimensional supermanifold as follows:

$\phi(x) \longrightarrow \tilde{\Phi}(x, \theta, \bar{\theta}), \quad v(x) \longrightarrow \tilde{V}(x, \theta, \bar{\theta})$,

$C(x) \longrightarrow F(x, \theta, \bar{\theta}), \quad \bar{C}(x) \longrightarrow \bar{F}(x, \theta, \bar{\theta})$,

$\lambda(x) \longrightarrow \tilde{\lambda}(x, \theta, \bar{\theta})$,

where $Z^{M}=\left(x^{\mu}, \theta, \bar{\theta}\right)$ is the superspace coordinate that parameterizes the above $(2,2)$-dimensional superfield where, in the spacetime bosonic pair $(x, t), t$ is the bosonic (evolution) parameter of the theory and the set $(\theta, \bar{\theta})$ is a pair of Grassmannian variables (with $\theta^{2}=\bar{\theta}^{2}=0$ and $\theta \bar{\theta}+\bar{\theta} \theta=$ $0)$. The gauge variable of the $2 \mathrm{D}$ effective theory is $\lambda(x)$ [i.e. $\left.\lambda(x)=\lambda_{0}(x)+\lambda_{1}(x)\right]$ which couples with the chiral bosonic field $\phi(x)$ and its orthogonal counterpart $\left[\lambda_{0}(x)-\lambda_{1}(x)\right]$ remains inert in our whole discussion. Thus, we define the appropriate 1-form gauge connection as

$$
\begin{aligned}
A^{(1)}= & \mathrm{d} x^{\mu} \lambda_{\mu} \equiv \frac{1}{2}\left(\mathrm{~d} x^{0}+\mathrm{d} x^{1}\right)\left(\lambda_{0}+\lambda_{1}\right) \\
& +\frac{1}{2}\left(\mathrm{~d} x^{0}-\mathrm{d} x^{1}\right)\left(\lambda_{0}-\lambda_{1}\right) \\
\equiv & \mathrm{d} x^{+}\left(\lambda_{0}+\lambda_{1}\right)+\mathrm{d} x^{-}\left(\lambda_{0}-\lambda_{1}\right) \equiv \mathrm{d} t \lambda,
\end{aligned}
$$

\footnotetext{
6 The WZ field $v$ is introduced by hand (i.e. $\phi \longrightarrow \phi-v, \lambda \longrightarrow \lambda+\dot{v}$ ) to convert the Lagrangian density (4) into (5) (modulo the gauge-fixing and FP ghost terms). The redefinition of $\phi$ gets rid of the WZ field $v$ from our theory at the quantum level.
}

where we have ignored the $\mathrm{d} x^{-}$component because of our preceding discussions and have identified the $\mathrm{d} x^{+}$component with the differential $\mathrm{d} t$ that corresponds to the evolution parameter of our theory. The exterior derivative $\mathrm{d}=\mathrm{d} t \partial_{t}$ (with $\mathrm{d}^{2}=0$ ) has been taken in our theory because of the above arguments. It is evident that $\mathrm{d} A^{(1)}=0$ due to $(\mathrm{d} t \wedge \mathrm{d} t)=0$. These 1 -forms of geometrical interest can be generalized onto our chosen $(2,2)$-dimensional supermanifold as

$\mathrm{d} \longrightarrow \tilde{\mathrm{d}}=\mathrm{d} Z^{M} \partial_{M} \equiv \mathrm{d} t \partial_{t}+\mathrm{d} \theta \partial_{\theta}+\mathrm{d} \bar{\theta} \partial_{\bar{\theta}}, \quad \tilde{\mathrm{d}}^{2}=0$,

$A^{(1)} \longrightarrow \tilde{A}^{(1)}=\mathrm{d} Z^{M} A_{M}$

$$
\equiv \mathrm{d} t \tilde{\lambda}(x, \theta, \bar{\theta})+\mathrm{d} \theta \bar{F}(x, \theta, \bar{\theta})+\mathrm{d} \bar{\theta} F(x, \theta, \bar{\theta}),
$$

where $\partial_{M}=\partial / \partial Z^{M} \equiv\left(\partial_{t}, \partial_{\theta}, \partial_{\bar{\theta}}\right)$ corresponds to the set of super-subspace partial derivatives corresponding to the $(1,2)$ dimensional super-subspace coordinates $(t, \theta, \bar{\theta})$ and $A_{M} \equiv$ $(\tilde{\lambda}(x, \theta, \bar{\theta}), F(x, \theta, \bar{\theta}), \bar{F}(x, \theta, \bar{\theta}))$ is the vector supermultiplet of superfields defined on the $(2,2)$-dimensional supermanifold, which is parametrized by $\left(x^{+}, x^{-}, \theta, \bar{\theta}\right)=$ $(t, x, \theta, \bar{\theta})$ in explicit components of the spacetime variables $(t, x)$ and Grassmannian variables $(\theta, \bar{\theta})$. We re-emphasize that the dynamics of our problem is such that we require only the superspace coordinates $(t, \theta, \bar{\theta})$. This is precisely the reason that we have the kind of generalizations (19), which are confined to $(1,2)$-dimensional super-submanifold only.

The superfields (17) can be expanded along the full Grassmannian directions (i.e., $1, \theta, \bar{\theta}, \theta \bar{\theta})$ of the full $(2,2)$ dimensional supermanifold as

$$
\begin{aligned}
\tilde{V}(x, \theta, \bar{\theta}) & =v(x)+i \theta \bar{F}_{2}(x)+i \bar{\theta} f_{2}(x)+i \theta \bar{\theta} b_{2}(x), \\
\tilde{\Phi}(x, \theta, \bar{\theta}) & =\phi(x)+i \theta \bar{F}_{1}(x)+i \bar{\theta} f_{1}(x)+i \theta \bar{\theta} b_{1}(x), \\
F(x, \theta, \bar{\theta}) & =C(x)+i \theta \bar{B}_{1}(x)+i \bar{\theta} B_{1}(x)+i \theta \bar{\theta} s(x), \\
\bar{F}(x, \theta, \bar{\theta}) & =\bar{C}(x)+i \theta \bar{B}_{2}(x)+i \bar{\theta} B_{2}(x)+i \theta \bar{\theta} \bar{s}(x), \\
\tilde{\lambda}(x, \theta, \bar{\theta}) & =\lambda(x)+\theta \bar{R}(x)+\bar{\theta} R(x)+i \theta \bar{\theta} S(x),
\end{aligned}
$$

where the secondary variables $\left(R, \bar{R}, s, \bar{s}, f_{1}, \bar{F}_{1}, f_{2}, \bar{F}_{2}\right)$ on the r.h.s. are fermionic and $\left(S, B_{1}, \bar{B}_{1}, B_{2}, \bar{B}_{2}, b_{1}, b_{2}\right)$ are the bosonic secondary fields, respectively. It is obvious that we have the basic fields of the effective theory $(\lambda, \phi, v, C, \bar{C})$, which are the limiting cases when we put $\theta=\bar{\theta}=0$. We shall derive the secondary fields in terms of the basic fields (as well as auxiliary fields) of the effective 2D theory by exploiting some physically motivated restrictions. First of all, let us exploit the horizontality condition (HC) which requires that $\tilde{\mathrm{d}} \tilde{A}^{(1)}=\mathrm{d} A^{(1)}=0$,

where the explicit form of the 1.h.s. (i.e. $\left.\tilde{\mathrm{d}} \tilde{A}^{(1)}\right)$ is

$$
\begin{aligned}
(\mathrm{d} t & \wedge \mathrm{d} \theta)\left[\partial_{t} \bar{F}-\partial_{\theta} \tilde{\lambda}\right]+(\mathrm{d} t \wedge \mathrm{d} \bar{\theta})\left[\partial_{t} F-\partial_{\bar{\theta}} \tilde{\lambda}\right] \\
& -(\mathrm{d} \theta \wedge \mathrm{d} \bar{\theta})\left[\partial_{\theta} F-\partial_{\bar{\theta}} \bar{F}\right]-(\mathrm{d} \theta \wedge \mathrm{d} \theta) \partial_{\theta} \bar{F} \\
& -(\mathrm{d} \bar{\theta} \wedge \mathrm{d} \bar{\theta}) \partial_{\bar{\theta}} F .
\end{aligned}
$$


Setting the coefficients of the differentials $(\mathrm{d} t \wedge \mathrm{d} \theta),(\mathrm{d} t \wedge$ $\mathrm{d} \bar{\theta}),(\mathrm{d} \theta \wedge \mathrm{d} \theta),(\mathrm{d} \theta \wedge \mathrm{d} \bar{\theta}),(\mathrm{d} \bar{\theta} \wedge \mathrm{d} \bar{\theta})$ equal to zero, we get the following relationships amongst the secondary fields and basic (as well as auxiliary) fields of our present theory:

$\bar{B}_{1}+B_{2}=0, \quad B_{1}=\bar{B}_{2}=0, \quad s=0$,

$R=\dot{C}, \quad \bar{R}=\dot{\bar{C}}, \quad S=\dot{B}, \quad \bar{s}=0$,

where we have used the expansions from (20). It is essential to point out that when we put the coefficients of $(\mathrm{d} \theta \wedge \mathrm{d} \bar{\theta})$ equal to zero, we obtain the Curci-Ferrari type of restriction $\bar{B}_{1}+B_{2}=0$, which is trivial for our simple theory. If we choose $B_{2}=B$ and $\bar{B}_{1}=-B$, we obtain the following explicit expansions for the superfields (20):

$$
\begin{aligned}
\tilde{\lambda}^{(h)}(x, \theta, \bar{\theta}) & =\lambda+\theta \dot{\bar{C}}+\bar{\theta} \dot{C}+\theta \bar{\theta}(i \quad \dot{B}) \\
& \equiv \lambda+\theta\left(s_{a b} \lambda\right)+\bar{\theta}\left(s_{b} \lambda\right)+\theta \bar{\theta}\left(s_{b} s_{a b} \lambda\right), \\
F^{(h)}(x, \theta, \bar{\theta}) & =C+\theta(-i B)+\bar{\theta}(0)+\theta \bar{\theta}(0) \\
& \equiv C+\theta\left(s_{a b} C\right)+\bar{\theta} s_{b}(C)+\theta \bar{\theta}\left(s_{b} s_{a b} C\right), \\
\bar{F}^{(h)}(x, \theta, \bar{\theta}) & =\bar{C}+\theta(0)+\bar{\theta}(i B)+\theta \bar{\theta}(0) \\
& \equiv \bar{C}+\theta\left(s_{a b} \bar{C}\right)+\bar{\theta}\left(s_{b} \bar{C}\right)+\theta \bar{\theta}\left(s_{b} s_{a b} \bar{C}\right),
\end{aligned}
$$

where the superscript $(h)$ stands for the expansion of the superfields after the application of HC. A close look at (24) establishes that we have already derived the following:

$$
\begin{aligned}
& s_{b} \lambda=\dot{C}, \quad s_{a b} \lambda=\dot{\bar{C}}, \quad s_{b} s_{a b} \lambda=i \dot{B}, \\
& s_{b} C=0, \quad s_{a b} C=-i B, \quad s_{b} s_{a b} C=0, \\
& s_{b} \bar{C}=i B, \quad s_{a b} \bar{C}=0, \quad s_{b} s_{a b} \bar{C}=0 .
\end{aligned}
$$

The requirement of nilpotency, in the above, implies that $s_{b} B=0$ and $s_{a b} B=0$.

To obtain the off-shell nilpotent and absolutely anticommuting (anti-)BRST symmetry transformations for $\phi(t)$ and $v(t)$ variables, we have to exploit the key ideas of the augmented version of the BT-superfield formalism [16-19] where we demand that all the (anti-)BRST-invariant quantities should remain independent of the "soul" coordinates (i.e., $\theta$ and $\bar{\theta}$ ) when they are generalized to the supermanifold. Thus, we invoke the following (anti-)BRST-invariant restrictions on the (super)fields:

$\tilde{\lambda}^{(h)}(x, \theta, \bar{\theta})+\dot{\tilde{\Phi}}(x, \theta, \bar{\theta})=\lambda(x)+\dot{\phi}(x)$,

$\tilde{\lambda}^{(h)}(x, \theta, \bar{\theta})+\dot{\tilde{V}}(x, \theta, \bar{\theta})=\lambda(x)+\dot{v}(x)$,

which have been taken due to our observations that $s_{(a) b}[\lambda(x)$ $+\dot{\phi}(x)]=0$ and $s_{(a) b}[\lambda(x)+\dot{v}(x)]=0$. We note that the (anti-)BRST-invariant restrictions (26) and HC are intertwined together because the expansion for $\tilde{\lambda}^{(h)}(x, \theta, \bar{\theta})$ has to be taken from (24), which has been derived after the application of the HC [i.e. $\tilde{\mathrm{d}} \tilde{A}^{(1)}=\mathrm{d} A^{(1)}=0$ ] in our theory.
Explicit substitution of (20) and (24) into (26) leads to the following relationships:

$f_{1}=i C, \quad \bar{F}_{1}=i \bar{C}, \quad b_{1}=-B$,

$f_{2}=i C, \quad \bar{F}_{2}=i \bar{C}, \quad b_{2}=-B$.

Thus, we have already obtained the expressions for the secondary fields in terms of the basic fields and auxiliary field $(B)$. Finally, we have the following expansions:

$$
\begin{aligned}
\tilde{\Phi}^{(b)}(x, \theta, \bar{\theta}) & =\phi+\theta(-\bar{C})+\bar{\theta}(-C)+\theta \bar{\theta}(-i B) \\
& \equiv \phi+\theta\left(s_{a b} \phi\right)+\bar{\theta}\left(s_{b} \phi\right)+\theta \bar{\theta}\left(s_{b} s_{a b} \phi\right), \\
\tilde{V}^{(b)}(x, \theta, \bar{\theta}) & =v+\theta(-\bar{C})+\bar{\theta}(-C)+\theta \bar{\theta}(-i B) \\
& \equiv v+\theta\left(s_{a b} v\right)+\bar{\theta}\left(s_{b} v\right)+\theta \bar{\theta}\left(s_{b} s_{a b} v\right),
\end{aligned}
$$

where the superscript $(b)$ stands for the expansions of the supervariables after the application of the nilpotent (anti-)BRST-invariant restrictions.

A close look at the above expansions yield the following (anti-)BRST symmetry transformations for the fields $\phi(t)$ and $v(t)$ :

$$
\begin{aligned}
& s_{b} \phi=-C, \quad s_{a b} \phi=-\bar{C}, \quad s_{b} s_{a b} \phi=-i B, \\
& s_{b} v=-C, \quad s_{a b} v=-\bar{C}, \quad s_{b} s_{a b} v=-i B .
\end{aligned}
$$

We draw the conclusion that we have already derived the full set of off-shell nilpotent and absolutely anticommuting (anti-)BRST symmetry transformations ${ }^{7}$ for the chiral bosonic system within the framework of the superfield approach to the BRST formalism ([f. (25), (29)]. We would like to close this section with the remark that there is a mapping between (anti-)BRST symmetry transformations and the translational generators along the Grassmannian directions of the full (2, 2)-dimensional supermanifold as can be seen from the following relationships:

$$
\begin{aligned}
& \left.\frac{\partial}{\partial \theta} \Omega^{(h, b)}(x, \theta, \bar{\theta})\right|_{\bar{\theta}=0}=s_{a b} \omega(x), \\
& \left.\frac{\partial}{\partial \bar{\theta}} \Omega^{(h, b)}(x, \theta, \bar{\theta})\right|_{\theta=0}=s_{b} \omega(x),
\end{aligned}
$$

where $\Omega^{(h, b)}(x, \theta, \bar{\theta})$ is the generic superfield [obtained after the application of the $\mathrm{HC}$ and (anti-)BRST invariant restrictions] and $\omega(x)$ is the generic field of the effective 2D system. It is evident that the nilpotency and absolute anticommutativity properties of the (anti-)BRST transformations $s_{(a) b}$ are intimately connected with such kinds of properties associated with the Grassmannian translational generators $\partial_{\theta}$ and $\partial_{\bar{\theta}}$ (i.e. $\partial_{\theta}^{2}=\partial_{\bar{\theta}}^{2}=0, \partial_{\theta} \partial_{\bar{\theta}}+\partial_{\bar{\theta}} \partial_{\theta}=0$ ).

\footnotetext{
7 The on-shell nilpotent version of the (anti-)BRST symmetry transformations can be also derived from the superfield approach if we replace the Nakanishi-Lautrup field by $-(\dot{\lambda}-v-\phi)$ [i.e. $B=-(\dot{\lambda}-v-\phi)]$.
} 


\section{4 (Anti-)BRST invariance, nilpotency, and anticommutativity: superfield approach}

In this section, we provide the geometrical basis for the existence of the nilpotency and absolute anticommutativity properties of the (anti-)BRST charges and the (anti-)BRST invariance of the Lagrangian density (5) (and the corresponding action) within the framework of the superfield formalism. Let us begin with the (anti-)BRST invariance of the action integral $S=\int \mathrm{d} x \int \mathrm{d} t \mathcal{L}_{b}$, illustrated in (7). Having obtained the expressions in (24) and (28), it can readily be seen that the Lagrangian density (5) can be generalized to the $(2,2)$ dimensional supermanifold in the following manner:

$$
\begin{aligned}
\mathcal{L}_{b} \longrightarrow \tilde{\mathcal{L}}_{b}= & \frac{1}{2}\left[\dot{\tilde{\Phi}}^{(b)} \dot{\tilde{\Phi}}^{(b)}-\dot{\tilde{V}}^{(b)} \dot{\tilde{V}}^{(b)}\right] \\
& +\dot{\tilde{V}}^{(b)}\left[\tilde{V}^{(b)}-\tilde{\Phi}^{(b)}\right]-\frac{1}{2}\left[\tilde{\Phi}^{\prime(b)}-\tilde{V}^{\prime(b)}\right]^{2} \\
& +\tilde{\lambda}^{(h)}\left[\dot{\tilde{\Phi}}^{(b)}-\dot{\tilde{V}}^{(b)}+\tilde{V}^{\prime(b)}-\tilde{\Phi}^{(b)}\right] \\
& +B(x)\left[\dot{\tilde{\lambda}}^{(h)}-\tilde{V}^{(b)}-\tilde{\Phi}^{(b)}\right]+\frac{B^{2}(x)}{2} \\
& -i \dot{\bar{F}}^{(h)} \dot{F}^{(h)}+2 i \bar{F}^{(h)} F^{(h)}
\end{aligned}
$$

where it is elementary to check that we have

$$
\begin{aligned}
& \tilde{V}^{\prime(b)}(x, \theta, \bar{\theta})-\tilde{\Phi}^{\prime(b)}(x, \theta, \bar{\theta})=v^{\prime}(x)-\phi^{\prime}(x), \\
& \dot{\tilde{\Phi}}^{(b)}(x, \theta, \bar{\theta})-\dot{\tilde{V}}^{(b)}(x, \theta, \bar{\theta})+\tilde{V}^{\prime(b)}(x, \theta, \bar{\theta}) \\
& \quad-\tilde{\Phi}^{\prime(b)}(x, \theta, \bar{\theta})=\dot{\phi}(x)-\dot{v}(x)+v^{\prime}(x)-\phi^{\prime}(x) .
\end{aligned}
$$

Now, it is straightforward to observe that

$$
\left.\frac{\partial}{\partial \bar{\theta}} \tilde{\mathcal{L}}_{b}\right|_{\theta=0}=\frac{\partial}{\partial t}[B \dot{C}],\left.\quad \frac{\partial}{\partial \theta} \tilde{\mathcal{L}}_{b}\right|_{\bar{\theta}=0}=\frac{\partial}{\partial t}[B \dot{\bar{C}}],
$$

which matches with (7) obtained in Sect. 2. It is worthwhile to mention that, in the above computation, we have first calculated all the $\theta$ and $\bar{\theta}$ dependent terms separately and then we have taken the derivatives with respect to $\bar{\theta}$ and $\theta$.

In view of the mapping (30), it is self-evident that we have obtained the following:

$$
\left.\frac{\partial}{\partial \bar{\theta}} \tilde{\mathcal{L}}_{b}\right|_{\theta=0} \longleftrightarrow s_{b} \mathcal{L}_{b},\left.\quad \frac{\partial}{\partial \theta} \tilde{\mathcal{L}}_{b}\right|_{\bar{\theta}=0} \longleftrightarrow s_{a b} \mathcal{L}_{b} .
$$

Geometrically, we have the following interpretation. The BRST and anti-BRST invariance of the Lagrangian density in the ordinary space can be explained by the shift transformations of the super Lagrangian density along the $\bar{\theta}$ and $\theta$ directions of the supermanifold, respectively, such that the outcome is a total spacetime derivative in the ordinary space. In other words, the physical (anti-)BRST invariance can be mathematically stated as the observation that the operation of the derivative w.r.t. Grassmannian variables (i.e. $\partial_{\bar{\theta}}$ and $\partial_{\theta}$ ) on the super Lagrangian density (31) produces the total time derivatives in the ordinary space.

Now we dwell on the proof of the nilpotency and anticommutativity of the (anti-)BRST charges within the framework of superfield formulation. First of all, it can be clearly checked that the (anti-)BRST charges can be expressed in terms of the expansions (24) and (28) in the following two equivalent forms:

$$
\begin{aligned}
Q_{a b} & =\int \mathrm{d} x\left[\left.\frac{\partial}{\partial \theta}\left[i\left(\bar{F}^{(h)} \dot{F}^{(h)}+F^{(h)} \dot{\bar{F}}^{(h)}\right)\right]\right|_{\bar{\theta}=0}\right] \\
& \equiv \int \mathrm{d} x\left[\left.\int \mathrm{d} \theta\left[i\left(\bar{F}^{(h)} \dot{F}^{(h)}+F^{(h)} \dot{\bar{F}}^{(h)}\right)\right]\right|_{\bar{\theta}=0}\right], \\
Q_{b} & =\int \mathrm{d} x\left[\left.\frac{\partial}{\partial \bar{\theta}}\left[-i\left(\bar{F}^{(h)} \dot{F}^{(h)}+F^{(h)} \dot{\bar{F}}^{(h)}\right)\right]\right|_{\theta=0}\right] \\
& \equiv \int \mathrm{d} x\left[\left.\int d \bar{\theta}\left[-i\left(\bar{F}^{(h)} \dot{F}^{(h)}+F^{(h)} \dot{\bar{F}}^{(h)}\right)\right]\right|_{\theta=0}\right] .
\end{aligned}
$$

In view of the mappings (30), (35) can be translated into the ordinary space in terms of the (anti-)BRST symmetry transformations as

$$
\begin{aligned}
Q_{a b} & =\int \mathrm{d} x s_{a b}[i(\bar{C} \dot{C}+C \dot{\bar{C}})], \\
Q_{b} & =\int \mathrm{d} x s_{b}[-i(\bar{C} \dot{C}+C \dot{\bar{C}})] .
\end{aligned}
$$

It is now elementary to prove that $\partial_{\theta} Q_{a b}=0, \partial_{\bar{\theta}} Q_{b}=0$ and $s_{a b} Q_{a b}=0, s_{b} Q_{b}=0$ [cf. (35), (36)]. These relationships provide a connection between the nilpotency property associated with $\partial_{\theta}{ }^{2}=\partial_{\bar{\theta}}{ }^{2}=0$ and $s_{(a) b}^{2}=0$. Furthermore, the definition of generators in terms of conserved charges $s_{b} Q_{b}=$ $-i\left\{Q_{b}, Q_{b}\right\}=0$ and $s_{a b} Q_{a b}=-i\left\{Q_{a b}, Q_{a b}\right\}=0$ prove the nilpotency of the charges $Q_{(a) b}$ in a subtle manner.

In view of expansions in (24) and (28), there are other ways to express the (anti-)BRST charges. These are explicitly written as follows:

$$
\begin{aligned}
& Q_{a b}=\int \mathrm{d} x\left[\frac{\partial}{\partial \bar{\theta}} \frac{\partial}{\partial \theta}\left(i \tilde{\lambda}^{(h)} \bar{F}^{(h)}\right)\right], \\
& Q_{a b}=\int \mathrm{d} x\left[\frac{\partial}{\partial \bar{\theta}}\left(i \dot{\bar{F}}^{(h)} \bar{F}^{(h)}\right)\right], \\
& Q_{a b}=\int \mathrm{d} x\left[\frac{\partial}{\partial \bar{\theta}} \frac{\partial}{\partial \theta}\left(-i \dot{\tilde{\Phi}}^{(b)} \bar{F}^{(h)}\right)\right], \\
& Q_{a b}=\int \mathrm{d} x\left[\frac{\partial}{\partial \bar{\theta}} \frac{\partial}{\partial \theta}\left(-i \dot{\tilde{V}}^{(b)} \bar{F}^{(h)}\right)\right], \\
& Q_{b}=\int \mathrm{d} x\left[\frac{\partial}{\partial \bar{\theta}} \frac{\partial}{\partial \theta}\left(i \tilde{\lambda}^{(h)} F^{(h)}\right)\right], \\
& Q_{b}=\int \mathrm{d} x\left[\frac{\partial}{\partial \theta}\left(-i \dot{F}^{(h)} F^{(h)}\right)\right], \\
& Q_{b}=\int \mathrm{d} x\left[\frac{\partial}{\partial \bar{\theta}} \frac{\partial}{\partial \theta}\left(-i \dot{\tilde{\Phi}}^{(b)} F^{(h)}\right)\right], \\
& Q_{b}=\int \mathrm{d} x\left[\frac{\partial}{\partial \bar{\theta}} \frac{\partial}{\partial \theta}\left(-i \dot{\tilde{V}}^{(b)} F^{(h)}\right)\right],
\end{aligned}
$$


which can be translated into the ordinary space in terms of symmetries $s_{(a) b}$ as follows:

$$
\begin{aligned}
Q_{a b} & =\int \mathrm{d} x\left[s_{b} s_{a b}(i \lambda \bar{C})\right], \\
Q_{a b} & =\int \mathrm{d} x\left[s_{b}(i \dot{\bar{C}} \bar{C})\right], \\
Q_{a b} & =\int \mathrm{d} x\left[s_{b} s_{a b}(-i \dot{\phi} \bar{C})\right], \\
Q_{a b} & =\int \mathrm{d} x\left[s_{b} s_{a b}(-i \dot{v} \bar{C})\right], \\
Q_{b} & =\int \mathrm{d} x\left[s_{b} s_{a b}(i \lambda C)\right], \\
Q_{b} & =\int \mathrm{d} x\left[s_{a b}(-i \dot{C} C)\right], \\
Q_{b} & =\int \mathrm{d} x\left[s_{b} s_{a b}(-i \dot{\phi} C)\right], \\
Q_{b} & =\int \mathrm{d} x\left[s_{b} s_{a b}(-i \dot{v} \bar{C})\right] .
\end{aligned}
$$

The above expressions prove the nilpotency and absolute anticommutativity in a very simple manner. This is due to the fact that $\partial_{\theta}{ }^{2}=\partial_{\bar{\theta}}{ }^{2}=0, \partial_{\theta} \partial_{\bar{\theta}}+\partial_{\bar{\theta}} \partial_{\theta}=0$. For instance, it can be seen that $Q_{b}=\int \mathrm{d} x s_{a b}(-i \dot{C} C)$ implies that $s_{a b} Q_{b}=0$ due to the nilpotency of $s_{a b}$. However, if we exploit the definition of the generator $s_{a b} Q_{b}=$ $-i\left\{Q_{b}, Q_{a b}\right\}$, we observe that the nilpotency of $s_{a b}$ (i.e. $\left.s_{a b}^{2}=0\right)$ is also connected with the absolute anticommutativity of the (anti-)BRST charges (i.e. $\left\{Q_{b}, Q_{a b}\right\}=0$ ). Similar explanations can be provided for all the other expressions for the (anti-)BRST charges which are listed in (38).

We close this section with the remarks that the nilpotency and absolute anticommutativity of the (anti-)BRST symmetry transformations $\left(s_{(a) b}^{2}=0, s_{b} s_{a b}+s_{a b} s_{b}=0\right)$ as well as the (anti-)BRST charges $\left(Q_{(a) b}^{2}=0, Q_{b} Q_{a b}+Q_{a b} Q_{b}=\right.$ $0)$ owe their origin to the key properties $\left(\partial_{\theta}{ }^{2}=\partial_{\bar{\theta}}{ }^{2}=\right.$ $\left.0, \partial_{\theta} \partial_{\bar{\theta}}+\partial_{\bar{\theta}} \partial_{\theta}=0\right)$ of the translational generators $\left(\partial_{\theta}, \partial_{\bar{\theta}}\right)$ along the Grassmannian directions $(\theta, \bar{\theta})$ of the supermanifold on which our theory is considered to be built. Furthermore, we note that the nilpotency and anticommutativity properties are intertwined together in a beautiful fashion within the framework of the augmented version of the BT-superfield formalism where one property depends on the other and vice versa.

\section{5 (Anti-)dual BRST symmetries: a brief synopsis}

The (anti-)BRST-invariant Lagrangian density (15) respects another set of off-shell nilpotent $\left(s_{(a) d}^{2}=0\right)$ and absolutely anticommuting $\left(s_{d} s_{a d}+s_{a d} s_{d}=0\right)$ symmetry transformations $\left(s_{(a) d}\right)$. These transformations are called the (anti-)dual BRST [or (anti-)co-BRST] symmetry transfor- mations because the gauge-fixing term ${ }^{8}(\dot{\lambda}-v-\phi)$ remains invariant under it. These transformations are listed, thus:

$$
\begin{aligned}
& s_{a d} \lambda=C, \quad s_{a d} \phi=\frac{1}{2} \dot{C}, \quad s_{a d} v=\frac{1}{2} \dot{C}, \\
& s_{a d} C=0, \quad s_{a d} \bar{C}=\frac{i}{2}\left(\dot{\phi}-\dot{v}+v^{\prime}-\phi^{\prime}\right), \\
& s_{a d}\left(\dot{\phi}-\dot{v}+v^{\prime}-\phi^{\prime}\right)=0, \\
& s_{d} \lambda=\bar{C}, \quad s_{d} \phi=\frac{1}{2} \dot{\bar{C}}, \quad s_{d} v=\frac{1}{2} \dot{\bar{C}}, \\
& s_{d} \bar{C}=0, \quad s_{d} C=-\frac{i}{2}\left(\dot{\phi}-\dot{v}+v^{\prime}-\phi^{\prime}\right), \\
& s_{d}\left(\dot{\phi}-\dot{v}+v^{\prime}-\phi^{\prime}\right)=0,
\end{aligned}
$$

where $s_{(a) d}$ are the (anti-)dual BRST symmetry transformations. One can explicitly check that the Lagrangian density (15) transforms to the total time derivatives as

$s_{d} \mathcal{L}_{b}^{(0)}=\frac{\partial}{\partial t}\left[\frac{\dot{\bar{C}}}{2}\left(\dot{\phi}-\dot{v}+v^{\prime}-\phi^{\prime}\right)\right]$,
$s_{a d} \mathcal{L}_{b}^{(0)}=\frac{\partial}{\partial t}\left[\frac{\dot{C}}{2}\left(\dot{\phi}-\dot{v}+v^{\prime}-\phi^{\prime}\right)\right]$.

The above observations demonstrate that the action integral $S=\int \mathrm{d} x \int \mathrm{d} t \mathcal{L}_{b}$ remains invariant under the transformations $s_{(a) d}$ for the physically meaningful fields that vanish off at infinity. According to Noether's theorem, the above continuous symmetry transformations lead to the derivation of the conserved charges as

$$
\begin{aligned}
Q_{a d} & =\int \mathrm{d} x\left[\frac{\dot{C}}{2}\left(\dot{\phi}-\dot{v}+v^{\prime}-\phi^{\prime}\right)-C(\dot{\lambda}-v-\phi)\right], \\
Q_{d} & =\int \mathrm{d} x\left[\frac{\dot{\bar{C}}}{2}\left(\dot{\phi}-\dot{v}+v^{\prime}-\phi^{\prime}\right)-\bar{C}(\dot{\lambda}-v-\phi)\right] .
\end{aligned}
$$

These charges are found to be nilpotent of order two (i.e. $\left.Q_{(a) d}^{2}=0\right)$ and they are also absolutely anticommuting $\left(Q_{d} Q_{a d}+Q_{a d} Q_{d}=0\right)$ in nature where the off-shoot $\left(\ddot{\phi}-\ddot{v}+\dot{v}^{\prime}-\dot{\phi}^{\prime}\right)+2(v+\phi-\dot{\lambda})=0$ of the EL equations of motion is used. ${ }^{9}$ This can be verified from $s_{d} Q_{a d}=$

\footnotetext{
${ }^{8}$ It can be checked that the 1D co-exterior derivative $\delta=+* \mathrm{~d} *$ (where $\mathrm{d}=\mathrm{d} t \partial_{t}$ ) operating on the 1 -form connection $A^{(1)}=\mathrm{d} t \lambda(x)$ produces $\dot{\lambda}(x)$ [because $\delta A^{(1)}=+* \mathrm{~d} * A^{(1)}=\dot{\lambda}(x)$ is a 0 -form]. One can add/subtract other 0 -forms $(\phi, v)$ fields to it as has been done in $(\dot{\lambda}-\phi-v)$.

9 The above charges (41) can be also expressed as $Q_{d}=\int \mathrm{d} x\left[\frac{\dot{\bar{C}}}{2}(\dot{\phi}-\right.$ $\left.\dot{v}+v^{\prime}-\phi^{\prime}\right)-\frac{\bar{C}}{2}\left(\ddot{\phi}-\ddot{v}+\dot{v}^{\prime}-\dot{\phi}^{\prime}\right], Q_{a d}=\int \mathrm{d} x\left[\frac{\dot{C}}{2}\left(\dot{\phi}-\dot{v}+v^{\prime}-\phi^{\prime}\right)-\right.$ $\frac{C}{2}\left(\ddot{\phi}-\ddot{v}+\dot{v}^{\prime}-\dot{\phi}^{\prime}\right]$ where we have to use $\left(\ddot{\phi}-\ddot{v}+\dot{v}^{\prime}-\dot{\phi}^{\prime}\right)+2(v+$ $\phi-\dot{\lambda})=0$ which emerges from the EL equations of motion, which are derived from the Lagrangian density (15). From these expressions, the anticommutativity property of $Q_{(a) d}$ becomes obvious and trivial because we can see explicitly (from the computations of the of 1.h.s. of $s_{a d} Q_{d}=-i\left\{Q_{a d}, Q_{d}\right\}=0$ and $\left.s_{d} Q_{a d}=-i\left\{Q_{d}, Q_{a d}\right\}=0\right)$ that it is true because the r.h.s. of both these relations turn out to be zero [due to the transformation properties of $Q_{(a) d}$ under the off-shell nilpotent (anti-)co-BRST transformations (39)].
} 
$-i\left\{Q_{a d}, Q_{d}\right\}=0$ and $s_{a d} Q_{d}=-i\left\{Q_{d}, Q_{a d}\right\}=0$ by computing the 1.h.s. of these relations by exploiting (39) and (41). We have discussed this issue in Appendix A, where we have differentiated between the (anti-)BRST and (anti-)coBRST symmetries of our present system and have pointed out some novel features.

With the ghost charge $Q_{g}=-i \int \mathrm{d} x[C \dot{\bar{C}}+\bar{C} \dot{C}]$, the off-shell nilpotent and absolutely anticommuting (anti-)coBRST charges obey the following algebra:

$Q_{d}^{2}=0, \quad Q_{a d}^{2}=0, \quad\left\{Q_{d}, Q_{a d}\right\}=0$,

$i\left[Q_{g}, Q_{d}\right]=-Q_{d}, \quad i\left[Q_{g}, Q_{a d}\right]=+Q_{a d}$,

which demonstrates that the ghost numbers of the (anti-)coBRST charges are +1 and -1 , respectively. Finally, we note that the conserved (anti-)co-BRST charges are the generators of continuous symmetry transformations listed in (39). In the forthcoming two sections, we shall derive the (anti-)coBRST symmetry transformations and prove the invariance of the Lagrangian density in the language of the superfield formalism (which captures the nilpotency as well as anticommutativity in a simple and straightforward manner).

\section{6 (Anti-)co-BRST symmetries: superfield approach}

To derive the (anti-)co-BRST symmetries (39), we shall exploit the key ingredients of the augmented version of the superfield approach to BRST formalism [16-19] where we shall demand that all the (anti-)co-BRST-invariant quantities should remain independent of the "soul" coordinates $\theta$ and $\bar{\theta}$ when the former are generalized onto the $(2,2)$-dimensional supermanifold. We observe that $s_{(a) d}[\phi-v]=0$. Thus, we demand that [cf. (20)]:

$\tilde{\Phi}(x, \theta, \bar{\theta})-\tilde{V}(x, \theta, \bar{\theta})=\phi(x)-v(x)$,

which immediately implies that

$\bar{f}_{1}=\bar{f}_{2} \equiv \bar{f}, \quad f_{1}=f_{2} \equiv f, \quad b_{1}=b_{2} \equiv b$.

We further note that $s_{(a) d}[\dot{\lambda}-\phi-v]=0$. This is due to the fact that the gauge-fixing term remains invariant under the (anti-)co-BRST symmetry transformations. Thus, we require the following restrictions on the (super)fields [cf. (20)]:

$$
\begin{aligned}
& \dot{\tilde{\lambda}}(x, \theta, \bar{\theta})-\tilde{\Phi}(x, \theta, \bar{\theta})-\tilde{V}(x, \theta, \bar{\theta}) \\
& \quad=\dot{\lambda}(x)-\phi(x)-v(x) .
\end{aligned}
$$

Taking the inputs from (44), we obtain the following relationships:

$$
\dot{\bar{R}}=2 i \bar{f}, \quad \dot{R}=2 i f, \quad \dot{S}=2 b .
$$

Furthermore, the relations (44) also imply the following relationships:

$$
\begin{aligned}
& \dot{\tilde{\Phi}}(x, \theta, \bar{\theta})-\dot{\tilde{V}}(x, \theta, \bar{\theta})+\tilde{V}^{\prime}(x, \theta, \bar{\theta})-\tilde{\Phi}^{\prime}(x, \theta, \bar{\theta}) \\
& \quad=\dot{\phi}(x)-\dot{v}(x)+v^{\prime}(x)-\phi^{\prime}(x),
\end{aligned}
$$

which is true because of the fact that $s_{(a) d}\left[\dot{\phi}-\dot{v}+v^{\prime}-\phi^{\prime}\right]=$ 0 . Thus, the equality in (47) is due to our basic ideology of the augmented version of the superfield formalism [16-19].

Now, we are in a position to exploit the mathematical potential of the (super) co-exterior derivatives in the application of the dual-horizontality condition, because we observe that $s_{(a) d}[\dot{\lambda}-2 \phi]=0$ and/or $s_{(a) d}[\dot{\lambda}-2 v]=0$. However, we also have seen that in ordinary 1D space, we have $\delta A^{(1)}=* \mathrm{~d} *[\mathrm{~d} t \lambda(t)]=\dot{\lambda}(t)$. Thus, we demand the following restriction under the dual-horizontality condition:

$\tilde{\delta} \tilde{A}^{(1)}-2 \tilde{\Phi}(x, \theta, \bar{\theta})=\delta A^{(1)}-2 \phi(x)$.

In Appendix B, we have explicitly computed the 1.h.s. and r.h.s. where $\tilde{\delta}=\star \tilde{d} \star, \tilde{d}=d t \partial_{t}+d \theta \partial_{\theta}+d \bar{\theta} \partial_{\bar{\theta}}$ and $\star$ is the Hodge-duality operation on the $(1,2)$-dimensional super-submanifold [of the general $(2,2)$-dimensional supermanifold] under consideration. Ultimately, our computations yield the following:

$\partial_{\theta} F=0 \quad \Longrightarrow \quad \bar{B}_{1}=0, \quad s=0$,

$\partial_{\bar{\theta}} \bar{F}=0 \quad \Longrightarrow \quad B_{2}=0, \quad \bar{s}=0$,

where we have taken the expansions from (20). The above values lead to the determination of the modified form of the superfields $F(x, \theta, \bar{\theta})$ and $\bar{F}(x, \theta, \bar{\theta})$ as

$F^{(r)}(x, \theta, \bar{\theta})=C(x)+i \bar{\theta} B_{1}(x)$,

$\bar{F}^{(r)}(x, \theta, \bar{\theta})=\bar{C}(x)+i \theta \bar{B}_{2}(x)$,

where the superscript $(r)$ stands for the reduced form of the superfields. The above reduced expansions should be used in our further discussions. Finally, we also have the following equality (see Appendix B for details):

$\dot{\tilde{\lambda}}+\left(\partial_{\bar{\theta}} F^{(r)}\right)+\left(\partial_{\theta} \bar{F}^{(r)}\right)-2 \tilde{\Phi}=\dot{\lambda}-2 \phi$,

which, ultimately, leads to the following relationships:

$B_{1}+\bar{B}_{2}=0 \quad \Longrightarrow \quad B_{1}=-\mathcal{B}=-\bar{B}_{2}$,

and the relations written in (46). The above choice, due to the dual-horizontality condition, leads to the following:

$$
\begin{aligned}
F^{(\mathrm{dh})}(x, \theta, \bar{\theta})= & C(x)+\theta(0)+\bar{\theta}(-i \mathcal{B}(x))+\theta \bar{\theta}(0) \\
\equiv & C(x)+\theta\left(s_{a d} C(x)\right)+\bar{\theta}\left(s_{d} C(x)\right) \\
& +\theta \bar{\theta}\left(s_{d} s_{a d} C(x)\right), \\
\bar{F}^{(\mathrm{dh})}(x, \theta, \bar{\theta})= & \bar{C}(x)+\theta(+i \mathcal{B}(x))+\bar{\theta}(0)+\theta \bar{\theta}(0) \\
\equiv & \bar{C}(x)+\theta\left(s_{a d} \bar{C}(x)\right)+\bar{\theta}\left(s_{d} \bar{C}(x)\right) \\
& +\theta \bar{\theta}\left(s_{d} s_{a d} \bar{C}(x)\right) .
\end{aligned}
$$


In explicit terms, we have already obtained the transformation properties of (anti-)ghost fields $(\bar{C}) \mathrm{C}$ under the (anti-)dual-BRST transformations,

$s_{d} C=-i \mathcal{B}, \quad s_{a d} C=0, s_{d} \mathcal{B}=0, s_{d} s_{a d} C=0$,

$s_{d} \bar{C}=0, s_{a d} \bar{C}=+i \mathcal{B}, s_{d} \mathcal{B}=0, s_{d} s_{a d} \bar{C}=0$,

where $s_{(a) d} \mathcal{B}=0$ has been obtained due to the requirement of the absolute nilpotency property (i.e. $s_{(a) d}^{2}=0$ ). We are free to choose $\mathcal{B}$ in terms of the basic fields so that the nilpotency and the absolute anticommutativity properties could be respected together. In this connection, it is worthwhile to point out that

$s_{(a) d}\left[\dot{\phi}-\dot{v}+v^{\prime}-\phi^{\prime}\right]=0$.

Thus, we choose ${ }^{10} \mathcal{B}=+\frac{1}{2}\left[\dot{\phi}-\dot{v}+v^{\prime}-\phi^{\prime}\right]$. In this choice, we have been guided by the fact that the field $\mathcal{B}$ should not explicitly depend on the gauge field $\lambda(t)$. This implies that we have the following:

$$
\begin{aligned}
F^{(\mathrm{dh})}(x, \theta, \bar{\theta})= & C(x)+\theta(0) \\
& +\bar{\theta}\left(-\frac{i}{2}\left[\dot{\phi}-\dot{v}+v^{\prime}-\phi^{\prime}\right]\right)+\theta \bar{\theta}(0), \\
\bar{F}^{(\mathrm{dh})}(x, \theta, \bar{\theta})= & \bar{C}(x)+\theta\left(+\frac{i}{2}\left[\dot{\phi}-\dot{v}+v^{\prime}-\phi^{\prime}\right]\right) \\
& +\bar{\theta}(0)+\theta \bar{\theta}(0),
\end{aligned}
$$

where the superscript (dh) on the superfields denotes the expansions obtained after the application of the dual-HC. In other words, we have derived the following (anti-)co-BRST transformations for the (anti-)ghost fields of our theory:

$s_{d} C=-\frac{i}{2}\left(\dot{\phi}-\dot{v}+v^{\prime}-\phi^{\prime}\right), \quad s_{a d} C=0$,

$s_{d} s_{a d} C=0$,

$s_{d} \bar{C}=0, \quad s_{a d} \bar{C}=+\frac{i}{2}\left(\dot{\phi}-\dot{v}+v^{\prime}-\phi^{\prime}\right)$,

$s_{d} s_{a d} \bar{C}=0$,

where the nilpotency $s_{(a) d}^{2}=0$ and the absolute anticommutativity property $\left(s_{d} s_{a d}+s_{a d} s_{d}\right) C=0$ and $\left(s_{d} s_{a d}+\right.$ $\left.s_{a d} s_{d}\right) \bar{C}=0$ are satisfied.

Finally, we note from the symmetry of the Lagrangian density (15) that $s_{(a) d}\left[\lambda\left(\dot{\phi}-\dot{v}+v^{\prime}-\phi^{\prime}\right)+2 i \bar{C} C\right]=0$. This observation leads to the requirement of the following equality:

$\tilde{\lambda}\left[\dot{\tilde{\Phi}}-\dot{\tilde{V}}+\tilde{V}^{\prime}-\tilde{\phi}^{\prime}\right]+2 i \bar{F}^{(\mathrm{dh})} \bar{F}^{(\mathrm{dh})}$

$=\lambda\left(\dot{\phi}-\dot{v}+v^{\prime}-\phi^{\prime}\right)+2 i \bar{C} C$.

\footnotetext{
${ }^{10}$ In Appendix $\mathrm{C}$, we provide a precise proof of this choice by using the basic ingredients of augmented superfield formalism where the (anti)co-BRST-invariant quantities are required to be independent of the Grassmannian variables when they are generalized to an appropriate supermanifold.
}

Using the expansions in (20), (56), and (47), we obtain the following:

$$
\bar{R}=+C, \quad R=+\bar{C}, \quad S=-\frac{1}{2}\left(\dot{\phi}-\dot{v}+v^{\prime}-\phi^{\prime}\right) .
$$

Now a comparison with (46) yields the following relationships between the secondary fields and other basic fields:

$f=-\frac{i}{2} \dot{\bar{C}}, \quad \bar{f}=-\frac{i}{2} \dot{C}, \quad b=-\frac{1}{4}\left(\ddot{\phi}-\ddot{v}+\dot{v}^{\prime}-\dot{\phi}^{\prime}\right)$.

The above relationships demonstrate that we have obtained all the secondary fields of the expansion in (20) in terms of the basic fields of the 2D ordinary theory. Collected together, these relationships are as follows:

$$
\begin{aligned}
& \bar{R}=+C, \quad R=+\bar{C}, \quad S=-\frac{1}{2}\left(\dot{\phi}-\dot{v}+v^{\prime}-\phi^{\prime}\right), \\
& f=-\frac{i}{2} \dot{\bar{C}}, \quad \bar{f}=-\frac{i}{2} \dot{C}, \quad b=-\frac{1}{4}\left(\ddot{\phi}-\ddot{v}+\dot{v}^{\prime}-\dot{\phi}^{\prime}\right) .
\end{aligned}
$$

The substitution of these values in the expansion (20) yields the following explicit expansions:

$$
\begin{aligned}
& \tilde{\lambda}^{(\mathrm{dh})}(x, \theta, \bar{\theta})=\lambda(x)+\theta(C)+\bar{\theta}(\bar{C}) \\
& \quad+\theta \bar{\theta}\left[-\frac{i}{2}\left(\dot{\phi}-\dot{v}+v^{\prime}-\phi^{\prime}\right)\right] \\
& \equiv \lambda(x)+\theta\left(s_{a d} \lambda\right)+\bar{\theta}\left(s_{d} \lambda\right)+\theta \bar{\theta}\left(s_{d} s_{a d} \lambda\right), \\
& F^{(\mathrm{dh})}(x, \theta, \bar{\theta})=C(x)+\theta(0)+\bar{\theta}\left(-\frac{i}{2}\left[\dot{\phi}-\dot{v}+v^{\prime}-\phi^{\prime}\right]\right) \\
& \quad+\theta \bar{\theta}(0) \\
& \equiv C(x)+\theta\left(s_{a d} C\right)+\bar{\theta}\left(s_{d} C\right)+\theta \bar{\theta}\left(s_{d} s_{a d} C\right), \\
& \bar{F}^{(\mathrm{dh})}(x, \theta, \bar{\theta})=\bar{C}(x)+\theta\left(\frac{i}{2}\left[\dot{\phi}-\dot{v}+v^{\prime}-\phi^{\prime}\right]\right)+\bar{\theta}(0) \\
& \quad+\theta \bar{\theta}(0) \\
& \equiv \bar{C}(x)+\theta\left(s_{a d} \bar{C}\right)+\bar{\theta}\left(s_{d} \bar{C}\right)+\theta \bar{\theta}\left(s_{d} s_{a d} \bar{C}\right), \\
& \tilde{\Phi}^{(\mathrm{as})}(x, \theta, \bar{\theta})=\phi(x)+\theta\left(+\frac{\dot{C}}{2}\right)+\bar{\theta}\left(+\frac{\dot{\bar{C}}}{2}\right) \\
& \quad+\theta \bar{\theta}\left(-\frac{i}{4} \frac{\partial}{\partial t}\left[\dot{\phi}-\dot{v}+v^{\prime}-\phi^{\prime}\right]\right) \\
& \equiv \phi(x)+\theta\left(s_{a d} \phi\right)+\bar{\theta}\left(s_{d} \phi\right)+\theta \bar{\theta}\left(s_{d} s_{a d} \phi\right), \\
& \tilde{V}^{(\text {as) }}(x, \theta, \bar{\theta})=v(x)+\theta\left(+\frac{\dot{\bar{C}}}{2}\right)+\bar{\theta}\left(+\frac{\dot{\bar{C}}}{2}\right) \\
& \quad+\theta \bar{\theta}\left(-\frac{i}{4} \frac{\partial}{\partial t}\left[\dot{\phi}-\dot{v}+v^{\prime}-\phi^{\prime}\right]\right) \\
& \equiv v(x)+\theta\left(s_{a d} v\right)+\bar{\theta}\left(s_{d} v\right)+\theta \bar{\theta}\left(s_{d} s_{a d} v\right),
\end{aligned}
$$

where the superscripts (as) and (dh) denote the expansions that have been obtained after the applications of the augmented superfield formalism and the dual-horizontality condition, respectively. A close look at the expansions (62) shows 
that we have already obtained the (anti-)co-BRST symmetries (39) for all the relevant fields of the theory.

We end this section with the remark that we also have the analog of the mapping (30) between the (anti-)co-BRST symmetry transformations $s_{(a) d}$ and the translational generators along the Grassmannian directions $(\theta, \bar{\theta})$ of the $(2$, 2)-dimensional supermanifold. This is succinctly expressed in the mathematical form as follows:

$$
\begin{aligned}
& \left.\frac{\partial}{\partial \theta} \Sigma^{(\mathrm{dh}, \mathrm{as})}(x, \theta, \bar{\theta})\right|_{\bar{\theta}=0}=s_{a d} \sigma(x), \\
& \left.\frac{\partial}{\partial \bar{\theta}} \Sigma^{(\mathrm{dh}, \mathrm{as})}(x, \theta, \bar{\theta})\right|_{\theta=0}=s_{d} \sigma(x),
\end{aligned}
$$

where the superscripts (dh, as) on the generic superfield $\Sigma^{(\mathrm{dh}, \mathrm{as})}(x, \theta, \bar{\theta})$ denote the expansions (for these fields) that have been obtained after the application of the dualhorizontality and the augmented version of the superfield restrictions and $\sigma(x)$ denotes the ordinary generic 2D field of the theory. It is obvious now that the nilpotency and the absolute anticommutativity of the (anti-)co-BRST symmetry transformations are encoded in such relations (i.e. $\left.\partial_{\theta}{ }^{2}=\partial_{\bar{\theta}}{ }^{2}=0, \partial_{\theta} \partial_{\bar{\theta}}+\partial_{\bar{\theta}} \partial_{\theta}=0\right)$ associated with the translational generators $\left(\partial_{\theta}, \partial_{\bar{\theta}}\right)$ along the Grassmannian directions $(\theta, \bar{\theta})$ of the $(2,2)$-dimensional supermanifold.

\section{7 (Anti-)co-BRST invariance, nilpotency, and anticommutativity: superfield approach}

In this section, we embark on the geometrical meaning of the (anti-)co-BRST invariance of the action integral [cf. (40)] and nilpotency and absolute anticommutativity of the (anti-)coBRST charges [cf. (42)] within the framework of our augmented superfield approach to the BRST formalism. First of all, we focus on the (anti-)co-BRST invariance of the action integral $S=\int \mathrm{d}^{2} x \mathcal{L}_{b}^{(0)}$ where $\mathcal{L}_{b}^{(0)}$ is the Lagrangian density (15) which respects the (anti-)co-BRST symmetries [as well as the on-shell nilpotent (anti-)BRST symmetries].

Taking the inputs from (62), it is straightforward to note that the Lagrangian density (15) can be generalized to the $(2,2)$-dimensional supermanifold in terms of the superfields obtained after application of the dual-horizontality condition and (anti-)co-BRST-invariant restrictions as [cf. (62)]:

$$
\begin{aligned}
\mathcal{L}_{b}^{(0)} \longrightarrow & \tilde{\mathcal{L}}_{b}^{(0)}=\frac{1}{2}\left[\dot{\tilde{\Phi}}^{(\mathrm{as})} \dot{\tilde{\Phi}}^{(\mathrm{as})}-\dot{\tilde{V}}^{(\mathrm{as})} \dot{\tilde{V}}^{(\mathrm{as})}\right] \\
& +\dot{\tilde{V}}^{(\mathrm{as})}\left[v^{\prime}-\phi^{\prime}\right]+\tilde{\lambda}^{(\mathrm{dh})}[\dot{\phi}-\dot{v}+\tilde{v}-\tilde{\phi}] \\
& -\frac{1}{2}\left[v^{\prime}-\phi^{\prime}\right]^{2}+B(x)\left[\dot{\tilde{\lambda}}^{(\mathrm{dh})}-\tilde{V}^{(\mathrm{as})}-\tilde{\Phi}^{(\mathrm{as})}\right] \\
& +\frac{B^{2}(x)}{2}-i \dot{\bar{F}}^{(\mathrm{dh})} \dot{F}^{(\mathrm{dh})}+2 i \bar{F}^{(\mathrm{dh})} F^{(\mathrm{dh})} \cdot(64)
\end{aligned}
$$

First of all, it is important to note that we have taken

$$
\begin{aligned}
\tilde{V}^{\prime(\text { as })}(x, \theta, \bar{\theta})-\tilde{\Phi}^{\prime(\text { as })}(x, \theta, \bar{\theta})= & v^{\prime}(x)-\phi^{\prime}(x), \\
\dot{\Phi}^{(\text {as })}-\dot{\tilde{V}}^{\text {(as) }}+\tilde{V}^{\prime(\text { as })}-\tilde{\Phi}^{\prime(\text { as })}= & \dot{\phi}(x)-\dot{v}(x) \\
& +v^{\prime}(x)-\phi^{\prime}(x),
\end{aligned}
$$

due to our observations in the super-expansions (62). Further, we observe that the Lagrangian density (15) has been generalized, in a straightforward manner, to our chosen (2, 2)-dimensional supermanifold just by replacing the ordinary 2D fields by their counterpart superfields, which we have been obtained after the application of the dual-horizontality condition and the (anti-)co-BRST-invariant restrictions on the supermanifold.

Now, we are in a position to capture the (anti-)co-BRST invariance (40) in terms of the geometrical quantities that are defined on the (2,2)-dimensional supermanifold. In other words, we have the following observations:

$$
\begin{aligned}
\left.\frac{\partial}{\partial \theta}\left[\tilde{\mathcal{L}}_{b}^{(0)}\right]\right|_{\bar{\theta}=0} & =s_{a d} \mathcal{L}_{b}^{(0)} \\
& \equiv \frac{\partial}{\partial t}\left[\frac{\dot{C}}{2}\left(\dot{\phi}-\dot{v}+v^{\prime}-\phi^{\prime}\right)\right], \\
\left.\frac{\partial}{\partial \bar{\theta}}\left[\tilde{\mathcal{L}}_{b}^{(0)}\right]\right|_{\theta=0} & =s_{d} \mathcal{L}_{b}^{(0)} \\
& \equiv \frac{\partial}{\partial t}\left[\frac{\dot{\bar{C}}}{2}\left(\dot{\phi}-\dot{v}+v^{\prime}-\phi^{\prime}\right)\right],
\end{aligned}
$$

which, in the ordinary 2D space, shows the (anti-)co-BRST invariance of the Lagrangian density (15). This is a consequence of the mapping we have obtained in (63). Geometrically, one can provide an interpretation for the superLagrangian density $\tilde{\mathcal{L}}_{b}^{(0)}$ as the sum of composite superfields, derived after the application of the dual-horizontality condition and (anti-)co-BRST invariant restrictions, such that its translations along the $\theta$ or $\bar{\theta}$ directions of the $(2,2)$ dimensional supermanifold leads to a total time derivative in the ordinary space. As a consequence, the action integral $\left(S=\int \mathrm{d}^{2} x \tilde{\mathcal{L}}_{b}^{(0)}\right)$ remains invariant under the nilpotent (anti-)co-BRST symmetry transformations in the ordinary space for the physically well-defined fields which vanish at infinity.

At this juncture, we concentrate on the geometrical interpretation of the nilpotency and the absolute anticommutativity of the (anti-)co-BRST charges $Q_{(a) d}$ in the language of the superfield approach to the BRST formalism. It can be checked that the (anti-)co-BRST charges can be written, in terms of the superfields (62), in two different ways:

$$
\begin{aligned}
Q_{d} & =\int \mathrm{d} x\left[\left.\frac{\partial}{\partial \bar{\theta}}\left(i \bar{F}^{(\mathrm{dh})} \dot{F}^{(\mathrm{dh})}+i F^{(\mathrm{dh})} \dot{\bar{F}}^{(\mathrm{dh})}\right)\right|_{\theta=0}\right] \\
& \equiv \int \mathrm{d} x\left[\left.\int \mathrm{d} \bar{\theta}\left(i \bar{F}^{(\mathrm{dh})} \dot{F}^{(\mathrm{dh})}+i F^{(\mathrm{dh})} \dot{\bar{F}}^{(\mathrm{dh})}\right)\right|_{\theta=0}\right],
\end{aligned}
$$




$$
\begin{aligned}
Q_{a d} & =\int \mathrm{d} x\left[\left.\frac{\partial}{\partial \theta}\left(i \bar{F}^{(\mathrm{dh})} \dot{F}^{(\mathrm{dh})}+i F^{(\mathrm{dh})} \dot{\bar{F}}^{(\mathrm{dh})}\right)\right|_{\bar{\theta}=0}\right] \\
& \equiv \int \mathrm{d} x\left[\left.\int \mathrm{d} \theta\left(i \bar{F}^{(\mathrm{dh})} \dot{F}^{(\mathrm{dh})}+i F^{(\mathrm{dh})} \dot{\bar{F}}^{(\mathrm{dh})}\right)\right|_{\bar{\theta}=0}\right] .
\end{aligned}
$$

The above expressions can be translated into our ordinary 2D space in terms of the nilpotent (anti-)co-BRST symmetry transformations $s_{(a) d}$ [in view of the mapping (63)] as follows:

$Q_{d}=i \int \mathrm{d} x s_{d}[C \dot{\bar{C}}+\bar{C} \dot{C}]$,

$Q_{a d}=i \int \mathrm{d} x s_{a d}[C \dot{\bar{C}}+\bar{C} \dot{C}]$,

which show the nilpotency of the (anti-)co-BRST charges due to $s_{(a) d}^{2}=0$. In the language of the superfield formalism, it is evident that $\partial_{\bar{\theta}} Q_{d}=0$ and $\partial_{\theta} Q_{a d}=0$ because of the nilpotency associated with $\partial_{\theta}^{2}=0, \partial_{\bar{\theta}}^{2}=0$. In other words, we have $s_{d} Q_{d}=-i\left\{Q_{d}, Q_{d}\right\}=0, s_{a d} Q_{a d}=$ $-i\left\{Q_{a d}, Q_{a d}\right\}=0$ due to the definition of the generators that imply $Q_{(a) d}^{2}=0$. We, ultimately, draw the conclusion that the nilpotency of the conserved (anti-)co-BRST charges is geometrically connected with the nilpotency property $\left(\partial_{\theta}^{2}=0, \partial_{\bar{\theta}}^{2}=0\right)$ of the translational generators along $(\theta, \bar{\theta})$-directions of our chosen (2,2)-dimensional supermanifold.

We are now in a position to provide the geometrical origin of the absolute anticommutativity properties of the transformations $s_{(a) d}$ (i.e. $s_{d} s_{a d}+s_{a d} s_{d}=0$ ) and nilpotent (anti-)coBRST charges $Q_{(a) d}$ (i.e. $Q_{d} Q_{a d}+Q_{a d} Q_{d}=0$ ). With this goal in mind, we observe

$Q_{a d}=\int \mathrm{d} x s_{d}(-i \dot{C} C), \quad Q_{d}=\int \mathrm{d} x s_{a d}(+i \dot{\bar{C}} \bar{C})$.

The above expressions, in the language of the symmetry generators, imply that $s_{d} Q_{a d}=-i\left\{Q_{a d}, Q_{d}\right\}=0$ and $s_{a d} Q_{d}=-i\left\{Q_{d}, Q_{a d}\right\}=0$ due to the off-shell nilpotency of $\left(s_{(a) d}^{2}=0\right)$ of the (anti-)co-BRST transformations. Thus, we note that the anticommutativity of the charges (i.e. $Q_{d} Q_{a d}+Q_{a d} Q_{d}=0$ ) is primarily connected with the nilpotency of the (anti-)co-BRST symmetry transformations. Due to the mapping (63), it is obvious that we can express the above charges as

$$
\begin{aligned}
Q_{a d} & =+\left.i \int \mathrm{d} x \frac{\partial}{\partial \bar{\theta}}\left[\dot{F}^{(\mathrm{dh})} F^{(\mathrm{dh})}\right]\right|_{\theta=0} \\
& \equiv+i \int \mathrm{d} x\left[\left.\int \mathrm{d} \bar{\theta}\left[\dot{F}^{(\mathrm{dh})} F^{(\mathrm{dh})}\right]\right|_{\theta=0},\right. \\
Q_{d} & =-\left.i \int \mathrm{d} x \frac{\partial}{\partial \theta}\left[\dot{\bar{F}}^{(\mathrm{dh})} \bar{F}^{(\mathrm{dh})}\right]\right|_{\bar{\theta}=0} \\
& \equiv-i \int \mathrm{d} x\left[\left.\int \mathrm{d} \theta\left[\dot{\bar{F}}^{(\mathrm{dh})} \bar{F}^{(\mathrm{dh})}\right]\right|_{\bar{\theta}=0},\right.
\end{aligned}
$$

which immediately lead to the observation that $\partial_{\bar{\theta}} Q_{a d}=0$ and $\partial_{\theta} Q_{d}=0$. Thus, we note that the absolute anticommutativity of the fermionic (anti-)co-BRST charges (i.e. $\left.\left\{Q_{d}, Q_{a d}\right\}=0\right)$ is connected with the nilpotency (i.e. $\left.\partial_{\theta}^{2}=0, \partial_{\bar{\theta}}^{2}=0\right)$ associated with the translational generators $\left(\partial_{\theta}, \partial_{\bar{\theta}}\right)$ along the Grassmannian directions of the $(2$, 2)-dimensional supermanifold.

There are other ways, too, for the (anti-)co-BRST symmetry to be expressed in terms of the geometrical quantities on the $(2,2)$-dimensional supermanifold. These are as follows:

$Q_{a d}=\int \mathrm{d} x\left[\frac{\partial}{\partial \bar{\theta}} \frac{\partial}{\partial \theta}\left(i \tilde{\lambda}^{(\mathrm{dh})} \dot{F}^{(\mathrm{dh})}\right)\right]$,

$Q_{a d}=\int \mathrm{d} x\left[\frac{\partial}{\partial \bar{\theta}} \frac{\partial}{\partial \theta}\left(-2 i \tilde{\Phi}^{(\mathrm{as})} F^{(\mathrm{dh})}\right)\right]$,

$Q_{a d}=\int \mathrm{d} x\left[\frac{\partial}{\partial \bar{\theta}} \frac{\partial}{\partial \theta}\left(-2 i \tilde{V}^{(\mathrm{as})} F^{(\mathrm{dh})}\right)\right]$,

$Q_{d}=\int \mathrm{d} x\left[\frac{\partial}{\partial \bar{\theta}} \frac{\partial}{\partial \theta}\left(i \tilde{\lambda}^{(\mathrm{dh})} \dot{\bar{F}}^{(\mathrm{dh})}\right)\right]$,

$Q_{d}=\int \mathrm{d} x\left[\frac{\partial}{\partial \bar{\theta}} \frac{\partial}{\partial \theta}\left(-2 i \tilde{\Phi}^{(\text {as })} \bar{F}^{(\mathrm{dh})}\right)\right]$,

$Q_{d}=\int \mathrm{d} x\left[\frac{\partial}{\partial \bar{\theta}} \frac{\partial}{\partial \theta}\left(-2 i \tilde{V}^{(\mathrm{as})} \bar{F}^{(\mathrm{dh})}\right)\right]$.

Due to the mapping (63), it is evident that we can express the above expansions in the ordinary 2D space of our BRSTinvariant gauge theory as

$Q_{a d}=\int \mathrm{d} x\left[s_{d} s_{a d}(i \lambda \dot{C})\right]$,

$Q_{a d}=\int \mathrm{d} x\left[s_{d} s_{a d}(-2 i \phi C)\right]$,

$Q_{a d}=\int \mathrm{d} x\left[s_{d} s_{a d}(-2 i v C)\right]$,

$Q_{d}=\int \mathrm{d} x\left[s_{d} s_{a d}(i \lambda \dot{\bar{C}})\right]$,

$Q_{d}=\int \mathrm{d} x\left[s_{d} s_{a d}(-2 i \phi \bar{C})\right]$,

$Q_{d}=\int \mathrm{d} x\left[s_{d} s_{a d}(-2 i v \bar{C})\right]$.

By exploiting the nilpotency $s_{(a) d}^{2}=0$ and anticommutativity ( $\left.s_{d} s_{a d}+s_{a d} s_{d}=0\right)$ of the (anti-)co-BRST symmetry transformations $s_{a(d)}$, it is clear that we can prove the nilpotency and the absolute anticommutativity of the (anti-)coBRST charges $Q_{a(d)}$ by exploiting the following definitions of the symmetry generators:

$s_{d} Q_{d}=-i\left\{Q_{d}, Q_{d}\right\}=0$,

$s_{d} Q_{a d}=-i\left\{Q_{d}, Q_{a d}\right\}=0$,

$s_{a d} Q_{d}=-i\left\{Q_{d}, Q_{a d}\right\}=0$,

$s_{a d} Q_{a d}=-i\left\{Q_{a d}, Q_{a d}\right\}=0$. 
If we look at the above charges in terms of superfields and the translational generators $\left(\partial_{\theta}, \partial_{\bar{\theta}}\right)$ [cf. (71)], it becomes crystal clear that the nilpotency and absolute anticommutativity of the (anti-)co-BRST charges (and corresponding transformations) are very intimately connected with such a kind of properties associated with the translational generators $\partial_{\theta}$ and $\partial_{\bar{\theta}}$. In other words, the properties $\left(\partial_{\theta}^{2}=0, \partial_{\bar{\theta}}^{2}=\right.$ $\left.0, \partial_{\theta} \partial_{\bar{\theta}}+\partial_{\bar{\theta}} \partial_{\theta}=0\right)$ are intertwined with the algebraic structures of the (anti-)co-BRST symmetry operators $s_{a(d)}$ and the corresponding conserved and nilpotent charges $Q_{a}(d)$.

\section{Conclusions}

The central results of our present endeavor are the precise derivations of the proper (i.e. nilpotent and absolutely anticommuting) (anti-)BRST and (anti-)co-BRST transformations for the 2D effective Lagrangian density [cf. (15)] of a chiral bosonic system at the quantum level. We have provided the geometrical basis for the above nilpotent symmetries in the language of the nilpotent (i.e. $\partial_{\theta}^{2}=0, \partial_{\bar{\theta}}^{2}=0$ ) translational generators $\left(\partial_{\theta}, \partial_{\bar{\theta}}\right)$ along the Grassmannian directions $(\theta, \bar{\theta})$ of our chosen $(2,2)$-dimensional supermanifold [parameterized by $Z^{M}=\left(x^{\mu}, \theta, \bar{\theta}\right)$ ] on which our present $2 \mathrm{D}$ ordinary field theory has been generalized (within the framework of our augmented version of the BT-superfield formalism, which is geometrically quite intuitive).

The dynamics of our present 2D theory is such that only a single component [cf. (4), (18)] of the 2D gauge field couples with the self-dual chiral bosonic field and its orthogonal component remains inert and does not participate in the dynamics of our present theory in any significant manner. This is a novel feature of a gauge theory, which we have never come across in our earlier discussions on the $p$-form ( $p=1,2,3$ ) gauge theories [25-29] within the framework of superfield and BRST formalisms. We have been able to capture this novel feature in the augmented version of our superfield approach to the BRST formalism and shown that the super-subspace variables $(t, \theta, \bar{\theta})$, parameterizing the $(1,2)$ dimensional super-submanifold, are good enough to capture the whole dynamics of the theory [despite the fact that the 2D ordinary theory is considered on the full $(2,2)$-dimensional supermanifold parameterized by the superspace variables $\left.Z^{M} \equiv\left(x^{\mu}, \theta, \bar{\theta}\right)\right]$.

The other novel feature of our present theory is the observation that the off-shell as well as on-shell nilpotent (anti-)BRST symmetries exist for the theory but only the off-shell nilpotent and absolutely anticommuting (anti-)coBRST symmetries are found to exist for the Lagrangian density (15) [which primarily respects only the on-shell nilpotent (anti-)BRST symmetry transformations]. It turns out that the off-shell nilpotent (anti-)co-BRST symmetry transformations (39) are absolutely anticommuting, too.
However, the corresponding (anti-)co-BRST charges $Q_{(a) d}$ are found to be absolutely anticommuting only when the offshoot [cf. (75)] of the EL equations of motion (76) [emerging from the Lagrangian density (15)] is utilized for its proof (see Appendix B for details).

It was a challenging task for us to check the sanctity for our proposal for the Hodge-duality $(\star)$ operation on a $(1,2)$-dimensional super-submanifold [of the full $(2,2)$ dimensional supermanifold on which our effective 2D theory has been generalized]. We have accomplished this goal (in Sect. 6) where we have exploited the Hodge-duality operation in the dual-horizontality condition [cf. (79), Appendix B] defined on the (1,2)-dimensional super-submanifold and derived the analog of the Curci-Ferrari restrictions [cf. (52)]. This exercise has enabled us to derive the correct off-shell nilpotent (anti-)co-BRST symmetry transformations of our theory. The idea of the dual-horizontality condition is a new ingredient in the realm of the superfield approach to the BRST formalism (which we have appropriately applied in the context of our present 2D theory).

We plan to apply the theoretical potential and power of the augmented version of our superfield formalism to supersymmetric gauge theories of phenomenological interest and establish a connection between the BRST type symmetries and supersymmetries. In particular, we wish to apply this method to obtain the precise (anti-)BRST symmetry transformations for the supersymmetric gauge theories. We have already obtained the supersymmetrization of the $\mathrm{HC}$ for a SUSY system of spinning relativistic particle and have obtained its proper (anti-)BRST symmetries and CF-type condition [30]. This direction of investigation is one of the open problems for us for our future endeavors.

Acknowledgments TB is grateful to BHU-fellowship and DS thanks UGC, Government of India, New Delhi, for financial support through RFSMS scheme under which the present investigation has been carried out. Fruitful comments by S. Krishna on some important issues of our present paper are thankfully acknowledged. We thank the reviewer for his enlightening comments.

Open Access This article is distributed under the terms of the Creative Commons Attribution License which permits any use, distribution, and reproduction in any medium, provided the original author(s) and the source are credited.

Funded by SCOAP $^{3}$ / License Version CC BY 4.0.

\section{Appendix A: On the (anti-)BRST and (anti-)co-BRST symmetry transformations}

One of the key observations of our present investigation is the fact that the Lagrangian density (5) and (15) respect the off-shell and on-shell nilpotent (anti-)BRST symmetries as quoted in (6) and (13), respectively. On the other hand, 
the Lagrangian density (15) respects the off-shell nilpotent (anti-)co-BRST symmetry transformations and there is no on-shell version of it. One of the characteristic and decisive features of these (anti-)co-BRST symmetry transformations is the requirement that the gauge-fixing term $(\dot{\lambda}-\phi-v)$, which basically owes its origin to the co-exterior derivative $(\delta=+* \mathrm{~d} *)$, remains invariant under the above infinitesimal, off-shell, and absolutely anticommuting symmetry transformations.

The interesting properties are the nilpotency and anticommutativity. It is straightforward to note that, for the (anti-)BRST symmetry transformations, the above sacrosanct properties are satisfied for the off-shell as well as onshell cases. This statement can be readily corroborated by (10) where the 1.h.s. of these relations can be computed with the help of (6) and (9) for the off-shell nilpotent (anti-)BRST symmetries. A similar exercise could also be performed for the on-shell nilpotent version. This, however, is not the case with the (anti-)co-BRST symmetries and their corresponding charges. In particular, in the case of the latter, it can be checked explicitly that the absolute anticommutativity of the (anti-)co-BRST charges,

$s_{d} Q_{a d}=-i\left\{Q_{a d}, Q_{d}\right\}=0$,

$s_{a d} Q_{d}=-i\left\{Q_{d}, Q_{a d}\right\}=0$,

can be true if and only if the equation

$\ddot{\phi}-\ddot{v}+\dot{v}^{\prime}-\dot{\phi}^{\prime}=2(\dot{\lambda}-v-\phi)$

is satisfied, which emerges from the appropriate combination of the following equations of motion that are derived from (15):

$\ddot{\lambda}=2 \dot{v}+\phi^{\prime}-v^{\prime}$,

$\ddot{v}-2 \dot{v}^{\prime}+\dot{\phi}^{\prime}+\left(\dot{\lambda}-\lambda^{\prime}\right)-\left(\phi^{\prime \prime}-v^{\prime \prime}\right)+(\dot{\lambda}-v-\phi)=0$,

$\ddot{\phi}+\left(\dot{\lambda}-\lambda^{\prime}\right)-\dot{v}^{\prime}-\left(\phi^{\prime \prime}-v^{\prime \prime}\right)-(\dot{\lambda}-v-\phi)=0$.

We would like to state, in passing, that the relation of type (75) is the analog of the celebrated Curci-Ferrari conditions for this system, which turns out to be an (anti-)co-BRSTinvariant quantity. In other words, we have $s_{(a) d}[(\ddot{\phi}-\ddot{v}+$ $\left.\left.\dot{v}^{\prime}-\dot{\phi}^{\prime}\right)-2(\dot{\lambda}-v-\phi)\right]=0$.

We close this appendix with the remark that, within the framework of the superfield approach to the BRST formalism, too, there is a key difference between the nilpotent (anti-)BRST and (anti-)co-BRST symmetries. In the former case, we have $\partial_{\theta} \bar{F}=0$ and $\partial_{\bar{\theta}} F=0$ when we set the coefficients of $(\mathrm{d} \theta \wedge \mathrm{d} \theta)$ and $(\mathrm{d} \bar{\theta} \wedge \mathrm{d} \bar{\theta})$ equal to zero in the HC (i.e. $\tilde{\mathrm{d}} \tilde{A}^{(1)}=\mathrm{d} A^{(1)}$ ) which leads to the relationships (23). On the other hand, in the latter case, we obtain the conditions $\partial_{\theta} F=0$ and $\partial_{\bar{\theta}} \bar{F}=0$ which lead to the relations
(49). The CF-type restrictions that emerge from these (dual-) horizontality conditions are [cf. (23), (52)]:

$\bar{B}_{1}+B_{2}=0, \quad B_{1}+\bar{B}_{2}=0$,

which enable us to obtain the absolute anticommutativity of the nilpotent (anti-)co-BRST and (anti-)BRST symmetry transformations, respectively.

\section{Appendix B: Hodge-duality operation on the (1,2)-dimen sional super-submanifold}

In this appendix, we demonstrate that we have the action of the Hodge-duality operation on a $(1,2)$-dimensional supersubmanifold [of the full $(2,2)$-dimensional supermanifold] in the following (super) 0-form relationship due to the dualhorizontality condition:

$\tilde{\delta} \tilde{A}^{(1)}-2 \tilde{\Phi}(x, \theta, \bar{\theta})=\delta A^{(1)}-2 \phi(x)$,

where $\delta=* \mathrm{~d} *$ and $\tilde{\delta}=\star \tilde{\mathrm{d}} \star$. Here the operator $*$ is the Hodge-duality operation on the 1D ordinary submanifold of the full 2D ordinary spacetime manifold (parameterized by $t$ ) and $\star$ is the Hodge-duality operation on the $(1,2)$-dimensional super-submanifold characterized by the supervariables $(t, \theta, \bar{\theta})$. It can be seen that the $\tilde{\delta} \tilde{A}^{(1)}=$ $+\star \tilde{\mathrm{d}} \star \tilde{A}^{(1)}$ can be computed from (19) in the following manner. First of all, we take the operation $\left(\star \tilde{A}^{(1)}\right)$ as given below in an explicit manner:

$$
\begin{aligned}
\star \tilde{A}^{(1)}= & \star[\mathrm{d} t \tilde{\lambda}(x, \theta, \bar{\theta})+\mathrm{d} \theta \bar{F}(x, \theta, \bar{\theta})+\mathrm{d} \bar{\theta} F(x, \theta, \bar{\theta})] \\
= & (\mathrm{d} \theta \wedge \mathrm{d} \bar{\theta}) \tilde{\lambda}(x, \theta, \bar{\theta})+(\mathrm{d} t \wedge \mathrm{d} \bar{\theta}) \bar{F}(x, \theta, \bar{\theta}) \\
& +(\mathrm{d} t \wedge \mathrm{d} \theta) F(x, \theta, \bar{\theta})
\end{aligned}
$$

which is nothing but a super 2-form on the (1,2)-dimensional superfield. Now the application $\tilde{\mathrm{d}}$ on (79) leads to the following super 3-form:

$$
\begin{aligned}
\tilde{d}\left(\star \tilde{A}^{(1)}\right)= & (\mathrm{d} t \wedge \mathrm{d} \theta \wedge \mathrm{d} \bar{\theta}) \dot{\tilde{\lambda}}+(\mathrm{d} t \wedge \mathrm{d} t \wedge \mathrm{d} \bar{\theta}) \dot{\bar{F}} \\
& +(\mathrm{d} t \wedge \mathrm{d} t \wedge \mathrm{d} \theta) \dot{F}+(\mathrm{d} \theta \wedge \mathrm{d} \theta \wedge \mathrm{d} \bar{\theta}) \partial_{\theta} \tilde{\lambda} \\
& -(\mathrm{d} \theta \wedge \mathrm{d} t \wedge \mathrm{d} \bar{\theta}) \partial_{\theta} \bar{F}-(\mathrm{d} \theta \wedge \mathrm{d} t \wedge \mathrm{d} \theta) \partial_{\theta} F \\
& +(\mathrm{d} \bar{\theta} \wedge \mathrm{d} \theta \wedge \mathrm{d} \bar{\theta}) \partial_{\bar{\theta}} \tilde{\lambda}-(\mathrm{d} \bar{\theta} \wedge \mathrm{d} t \wedge \mathrm{d} \bar{\theta}) \partial_{\bar{\theta}} \bar{F} \\
& -(\mathrm{d} \bar{\theta} \wedge \mathrm{d} t \wedge \mathrm{d} \theta) \partial_{\bar{\theta}} F
\end{aligned}
$$

In the above, the second and the third terms in the first line should be zero because $(\mathrm{d} t \wedge \mathrm{d} t)=0$. The first terms of the second and the third lines would be zero according to the prescription laid down in [31] because, on a $(1,2)$-dimensional superfield, a super 3 -form with merely $(\theta, \bar{\theta})$ terms cannot be defined (see, e.g., [31] for details). Thus, ultimately, we have 
the following:

$$
\begin{aligned}
\tilde{d}\left(\star \tilde{A}^{(1)}\right)= & (\mathrm{d} t \wedge \mathrm{d} \theta \wedge \mathrm{d} \bar{\theta}) \dot{\tilde{\lambda}}+(\mathrm{d} t \wedge \mathrm{d} \theta \wedge \mathrm{d} \bar{\theta}) \partial_{\theta} \bar{F} \\
& +(\mathrm{d} t \wedge \mathrm{d} \theta \wedge \mathrm{d} \theta) \partial_{\theta} F+(\mathrm{d} t \wedge \mathrm{d} \bar{\theta} \wedge \mathrm{d} \bar{\theta}) \partial_{\bar{\theta}} \bar{F} \\
& +(\mathrm{d} t \wedge \mathrm{d} \theta \wedge \mathrm{d} \bar{\theta}) \partial_{\bar{\theta}} F
\end{aligned}
$$

where we have used $(\mathrm{d} t \wedge \mathrm{d} \theta)=-(\mathrm{d} \theta \wedge \mathrm{d} t)$ and $(\mathrm{d} t \wedge$ $\mathrm{d} \bar{\theta})=-(\mathrm{d} \bar{\theta} \wedge \mathrm{d} t)$. Now, we are at a stage, to apply the final $\star$ on (81). Using the rules of [31], we have the following operation on the 3 -form differentials of the (1,2)-dimensional super-submanifold:

$\star(\mathrm{d} t \wedge \mathrm{d} \theta \wedge \mathrm{d} \bar{\theta})=1, \quad \star(\mathrm{d} t \wedge \mathrm{d} \theta \wedge \mathrm{d} \theta)=S^{\theta \theta}$,

$\star(\mathrm{d} t \wedge \mathrm{d} \bar{\theta} \wedge \mathrm{d} \bar{\theta})=S^{\bar{\theta} \bar{\theta}}$,

where $S^{\theta \theta}$ and $S^{\bar{\theta} \bar{\theta}}$ are symmetric so that if we take another $\star$ on (82) we should get back the original 3-form differentials (modulo a \pm sign). It is evident that all the super 0 -forms in (82) are independent of one another.

Taking the above into account, we have the following equation:

$\star \tilde{d} \star \tilde{A}^{(1)}=\left(\dot{\tilde{\lambda}}+\partial_{\theta} \bar{F}+\partial_{\bar{\theta}} F\right)+S^{\theta \theta} \partial_{\theta} F+S^{\bar{\theta} \bar{\theta}} \partial_{\bar{\theta}} \bar{F}$.

It is now obvious that the equality (78) can be expanded as follows:

$$
\begin{aligned}
& \left(\dot{\tilde{\lambda}}+\partial_{\theta} \bar{F}+\partial_{\bar{\theta}} F-2 \tilde{\Phi}\right)+S^{\theta \theta} \partial_{\theta} F+S^{\bar{\theta} \bar{\theta}} \partial_{\bar{\theta}} \bar{F} \\
& \quad=\dot{\lambda}-2 \phi .
\end{aligned}
$$

This is the explicit expression that has been taken in the main body of our text where we have computed the (anti-)dual BRST symmetry transformations for the (anti-)ghost fields of our theory in a correct fashion [cf. (53)].

\section{Appendix C: On the specific choice of $\mathcal{B}$}

In this appendix, we provide the precise derivation of the ad hoc choice made in Sect. 6 [cf. the comment after (55)] for the auxiliary variable

$\mathcal{B}=\frac{1}{2}\left(\dot{\phi}-\dot{v}+v^{\prime}-\phi^{\prime}\right)$,

from which ensues the relations $R=\bar{C}, \bar{R}=C, f=$ $-\frac{i}{2} \dot{\bar{C}}, \bar{f}=-\frac{i}{2} \dot{C}, b=-\frac{1}{4}\left(\ddot{\phi}-\ddot{v}+\dot{v}^{\prime}-\dot{\phi}^{\prime}\right)$ in the main body of our text (cf. Sect. 6). To derive these explicitly, we have to take recourse to the augmented version of supervariable approach where all the (anti-)BRST- [and/or (anti-)coBRST-] invariant quantities (of physical interest) are required to be independent of the Grassmannian variables $\theta$ and $\bar{\theta}$ when they are generalized to an appropriate supermanifold.

According to the above logic, we observe that the following additional quantities $\left(Q_{i}, i=1,2,3,4\right)$ of interest:

$Q_{1}=\lambda C, \quad Q_{2}=\phi \dot{C}, \quad Q_{3}=\lambda \bar{C}, \quad Q_{4}=\phi \dot{\bar{C}}$,

remain invariant under the nilpotent (anti-)co-BRST symmetry transformations $s_{(a) d}$ (i.e. $s_{a d} Q_{1}=s_{a d}[\lambda C]=$ $0, s_{a d} Q_{2}=s_{a d}[\phi \dot{C}]=0, s_{d} Q_{3}=s_{d}[\lambda \bar{C}]=$ $\left.0, s_{d} Q_{4}=s_{d}[\phi \dot{\bar{C}}]=0\right)$. Thus, we demand that the following equalities should hold true:

$$
\begin{aligned}
& \tilde{\lambda}(x, \theta, \bar{\theta}) \bar{F}^{(\mathrm{dh})}(x, \theta, \bar{\theta})=\lambda(x) \bar{C}(x), \\
& \tilde{\lambda}(x, \theta, \bar{\theta}) F^{(\mathrm{dh})}(x, \theta, \bar{\theta})=\lambda(x) C(x), \\
& \tilde{\Phi}(x, \theta, \bar{\theta}) \dot{\bar{F}}^{(\mathrm{dh})}(x, \theta, \bar{\theta})=\phi(x) \dot{\bar{C}}(x), \\
& \tilde{\Phi}(x, \theta, \bar{\theta}) \dot{F}^{(\mathrm{dh})}(x, \theta, \bar{\theta})=\phi(x) \dot{C}(x),
\end{aligned}
$$

which lead to the following relationships:

$$
\begin{aligned}
& i \lambda \mathcal{B}+\bar{R} \bar{C}=0, \quad R \bar{C}=0, \quad R \mathcal{B}+S \bar{C}=0, \\
& i \lambda \mathcal{B}-R C=0, \quad \bar{R} C=0, \quad \bar{R} \mathcal{B}+S C=0, \\
& \phi \dot{\mathcal{B}}+\bar{f} \dot{\bar{C}}=0, \quad \bar{f} \dot{C}=0, \quad i b \dot{\bar{C}}-f \dot{\mathcal{B}}=0, \\
& \phi \dot{\mathcal{B}}-f \dot{C}=0, \quad f \dot{\bar{C}}=0, \quad i b \dot{C}-\bar{f} \dot{\mathcal{B}}=0 \text {. }
\end{aligned}
$$

The above equations automatically show that we have $R \propto$ $\bar{C}, f \propto \dot{\bar{C}}, \bar{R} \propto C$ and $\bar{f} \propto \dot{C}$. We make one of the simplest choices: $R=\bar{C}, \bar{R}=C$. This choice is good enough to lead to all the values written in (61) (cf. Sect. 6). Thus, we observe that we have derived all the secondary variables in terms of the basic and auxiliary variables of the theory, which fully lead to the expansions (62) that incorporate all the transformations $s_{(a) d}$.

\section{References}

1. N. Marcus, J. Schwarz, Phys. Lett. B 115, 111 (1982)

2. D.J. Gross, J.A. Harvey, E. Martinec, R. Rohm, Phys. Rev. Lett. 54, $502(1985)$

3. X.G. Wen, Phys. Rev. Lett. 64, 2206 (1990)

4. M. Stone, Phys. Rev. B 41, 212 (1990)

5. J.V. Jose, L.P. Kadanoff, S. Kirkpatrik, D. Nelson, Phy. Rev. B 16, $1217(1977)$

6. P. Weigman, J. Phys. C 11, 1583 (1987)

7. D. Boyanovsky, R. Holman, Nucl. Phys. B 332, 641 (1990)

8. R. Floreanini, R. Jackiw, Phys. Rev. Lett. 59, 1873 (1987)

9. W. Siegel, Nucl. Phys. B 238, 307 (1984)

10. C. Imbimbo, A. Schwimmer, Phys. Lett. B 193, 455 (1987)

11. J.M.F. Labastida, M. Pernici, Phys. Rev. Lett. 59, 2511 (1987)

12. P.P. Srivastava, Phys. Rev. Lett. 63, 2791 (1989)

13. S. Upadhyay, B.P. Mandal, Eur. Phys. J. C 71, 1759 (2011)

14. L. Bonora, M. Tonin, Phys. Lett. B 98, 48 (1981) 
15. L. Bonora, P. Pasti, M. Tonin, Nuovo Cim. A 63, 353 (1981)

16. R.P. Malik, Eur. Phys. J. C 47, 219 (2006)

17. R.P. Malik, Eur. Phys. J. C 48, 825 (2006)

18. R.P. Malik, Eur. Phys. J. C 51, 169 (2007)

19. R.P. Malik, J. Phys. A 40, 4877 (2007)

20. G. Curci, R. Ferrari, Phys. Lett. B 63, 91 (1976)

21. P.P. Srivastava, Phys. Lett. B 234, 93 (1990)

22. P. Pasti, D. Sorokin, M. Tonin, Phys. Rev. D 55, 6292 (1997)

23. P. Pasti, D. Sorokin, M. Tonin. arXiv:9509052 [hep-th] (presented at conference C95-07-10.6)

24. U. Kulshreshtha, D.S. Kulshreshthra, H.J.W. Mueuller-Kirsten, Z. Phys. C 60, 427 (1993)

25. R.P. Malik, J. Phys. A 34, 4167 (2001)

26. R.P. Malik, Int. J. Mod. Phys. A 22, 3521 (2007)
27. S. Gupta, R.P. Malik, Eur. Phys. J. C 58, 517 (2008)

28. R. Kumar, S. Krishna, A. Shukla, R.P. Malik, Eur. Phys. J. C 72, 1980 (2012)

29. R. Kumar, S. Krishna, A. Shukla, R. P. Malik. arXiv:1203.5519 [hep-th] (to appear brief review in Int. J. Mod. Phys. A, 2014)

30. A. Shukla, S. Krishna, R.P. Malik, Eur. Phys. J. C 72, 2188 (2012)

31. R.P. Malik, Int. J. Mod. Phys. A 21, 3307 (2006) 